\title{
Ableitung von Handlungsoptionen
}

\author{
Coordinating Lead Authors (CLAs) \\ Ulrike Pröbstl-Haider \\ Lead Authors (LAs) \\ Ulrike Pröbstl-Haider, Nina Mostegl, Andrea Damm
}

Contributing Authors (CAs)

Franz Prettenthaler, Dagmar Lund-Durlacher, Astrid Gühnemann, Robert Steiger, Marc Olefs, Herbert Formayer, Claudia Hödl, Christoph Neger

\subsection{Grundsätzliche Strategien und Handlungsoptionen für einen „Paris Lifestyle“}

Bevor im Einzelnen auf die grundsätzlichen Strategien und Handlungsoptionen eingegangen wird, ist es wichtig, die dem Bericht zugrunde liegende Vision zu verdeutlichen.

Der Tourismus unterscheidet sich von anderen Wirtschaftszweigen dadurch, dass die Kunden wesentlich flexibler sind und sehr rasch - oftmals von einer Saison auf die

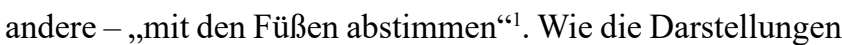
in Kap. 1 zeigen, hat diese Abhängigkeit durch eine Zunahme kürzerer Reisen und der sogenannten Stand-by-Touristen ${ }^{2}$ eher noch zugenommen. Daher kommt es im Tourismus nicht nur darauf an, ob und inwieweit das Angebot der österreichischen Tourismusbranche klimagerecht ist, sondern auch ob und wieweit es gelingt, den Kunden gezielt zu beeinflussen (Njoroge 2015).

$\mathrm{Zu}$ den grundsätzlichen Handlungsstrategien gehört dabei zunächst die Vision, die sich unter dem Begriff „Paris Lifestyle“3 einprägsam zusammenfassen lässt. Paris Lifestyle bedeutet in diesem Kontext auch im Urlaub und auf Reisen einen Lebensstil zu wählen, der dazu beiträgt, die in Paris vereinbarten Klimaziele einzuhalten. Der darin enthaltene positiv besetzte Bezug zu einem attraktiven Lebensstil, einer besonderen Lebensart, Kultur und Küche, für die Paris auch steht, ist beabsichtigt und verankert die wesentliche Zielsetzung, auf die es bei der Entwicklung von touristischen Produkten und deren Kommunikation

\footnotetext{
1 „Mit den Füßen abstimmen“ bedeutet eine andere Destination für den Urlaub aufzusuchen.

2 Als Stand-by-Tourist werden, in Anlehnung an die Stand-by-Funktion von technischen Geräten, Personen bezeichnet, die jederzeit reisebereit sind.

${ }^{3}$ Der Begriff „Paris Lifestyle“ leitet sich von dem Ort des internationalen Klimaabkommens ab.
}

ankommt: Es geht um einen anderen Lebensstil. Es geht darum, den Klimawandel bei der Buchung, bei der Anreise, bei der Gastronomie, der Produktentwicklung, aber auch bei betrieblichen Erneuerungsmaßnahmen usw. mitzudenken und aktiv zu werden.

Diese Handlungsstrategie zielt darauf ab, dass ein Urlaub in Österreich mit gutem Gewissen gebucht und angetreten werden kann. Das touristische Angebot soll zum Inbegriff eines Paris Lifestyles werden: ein genussvoller Vorzeigeurlaub und, über alle unterschiedlichen Angebote hinweg, ein Urlaub, der durch Erlebnisreichtum und durch Vermeidungs- und Klimawandelanpassungsmaßnahmen beeindruckt. Urlaub in Österreich könnte dazu einladen, diesen erlebten Lifestyle mit nach Hause zu nehmen und damit auch den Alltag der Gäste über den Urlaub hinaus positiv zu beeinflussen.

Die Einhaltung der Pariser Klimaziele unter Berücksichtigung ökonomischer, sozialer, politischer und technologischer Realitäten kann nur gelingen, wenn die dringend notwendige Reduktion von Treibhausgasen (THG) zu einem Projekt für alle wird, das alle relevanten Zielgruppen berücksichtigt und einen Paris Lifestyle zu einer attraktiven Art der Lebensgestaltung werden lässt (Schwarzinger et al. 2018). Bei einer konsumbasierten Emissionsbilanz in Österreich von rund 15 Tonnen $\mathrm{CO}_{2}$-Äquivalenten pro Kopf und Jahr (Windsperger et al. 2017) und einem Zielwert von nahe null im Jahr 2050 ergibt sich ein steiler Reduktionspfad, der aus heutiger Sicht ebenso ambitioniert wie alternativlos ist.

$\mathrm{Zu}$ den Vermeidungs- und Anpassungsstrategien und -maßnahmen ergeben sich verschiedene Ansatzpunkte (in Anlehnung an Njoroge 2015): 
- Anpassungs- und Vermeidungsmaßnahmen durch den Reisenden (Consumer Adaptation),

- Anpassungs- und Vermeidungsmaßnahmen durch den touristischen Betrieb (Business Adaptation),

- Anpassungs- und Vermeidungsmaßnahmen durch die regionale Destination (Destinationsadaptation),

- Anpassungs- und Vermeidungsmaßnahmen durch die Destination Österreich (National Destination Policy and Adaptation).

Diese spezifischen Ansätze werden nachstehend bezogen auf Österreich und die verschiedenen Bestandteile einer Reise beschrieben. Allerdings muss an dieser Stelle auch hervorgehoben werden, dass es sich bei der vorliegenden Studie in erster Linie um einen Sachstandsbericht handelt und daher nur grundsätzliche Lösungsansätze, nicht jedoch konkrete Maßnahmen erarbeitet werden konnten. Wir hoffen jedoch, dass die Handlungsoptionen in diesem Kapitel die Forschung und Forschungsförderung stimulieren werden.

\subsection{Handlungsebenen und spezifische Lösungsansätze}

\subsubsection{Anpassungs- und Vermeidungsmaßnahmen durch den Reisenden}

Im Zusammenhang mit der Klimawandelanpassung, insbesondere den zu treffenden Maßnahmen, wurde in der Vergangenheit vielfach die betriebliche Ebene in den Mittelpunkt gestellt (APCC 2014; EIW 2014; BMWFW et al. 2015). Daher soll hier, zu Beginn der Strategien, Herausforderungen und Lösungsansätze, zunächst nicht die Angebots-, sondern die Nachfrageseite in den Mittelpunkt gestellt werden. Deren Bedeutung wurde bereits in den vorausgegangenen Kapiteln erläutert: In Österreich waren im Kalenderjahr 2018 rund 110,4 Mio. Übernachtungen ausländischer und 39,4 Mio. Übernachtungen inländischer Gäste zu verzeichnen (Statistik Austria 2019), die während ihres Aufenthalts Dienstleistungen wie Zimmerservice oder Reinigungsdienste in Anspruch genommen und grob überschlagen rund 450 Mio. Mahlzeiten konsumiert haben (siehe Abschn. 5.1). Diese Zahlen verdeutlichen, wie hoch das Potenzial für Energieeinsparungen und die Reduzierung der Treibhausgasemissionen ist, wenn die Nachfrageseite in die Anpassungsstrategien aktiv mit aufgenommen wird. Alleine eine Änderung der Ernährungsgewohnheiten könnte zu einer Verringerung der Treibhausgasemissionen um bis zu 40 \% führen (Gössling und Peeters 2015).

\section{Die Reisenden informieren}

Einigkeit besteht darin, dass bewusstseinsbildende Maßnahmen sinnvoll sein können, um Touristinnen und Tou- risten in ihrer Urlaubsplanung und -umsetzung im Sinne des Klimaschutzes positiv zu beeinflussen (z. B. Wahl des Urlaubsortes, Wahl des Verkehrsmittels). Die gegenwärtig schlechte Verfügbarkeit von Informationen über die Klimabilanz von Produkten und Dienstleistungen führt dazu, dass selbst umweltbewusste und handlungsbereite Personen kaum in der Lage sind, eine informierte Konsumentscheidung zu treffen. Das bedeutet, dass über die Treibhausgasbilanz von Produkten und Dienstleistungen mehr als bisher Transparenz hergestellt werden muss, um eine faire Grundlage für die Internalisierung ${ }^{4}$ externer Kosten zu schaffen und um klimabewussten Konsumentinnen und Konsumenten eine informierte Entscheidung zu ermöglichen.

Dies bedeutet auch, dass die Informationsmöglichkeiten für die Reisenden zum Beispiel in Bezug auf Reisebüros, große Reiseanbieter und Buchungsplattformen, wie Booking. com oder Trivago, erheblich verbessert werden müssen. Dies kann etwa durch Ausweisung eines $\mathrm{CO}_{2}$-Fußabdruckes für die jeweiligen Angebote sowie durch Darstellungen von Zertifizierungen oder durch ein spezielles ECO-Labeling erreicht werden. In diesem Zusammenhang kann die zunehmende Bedeutung des Internets als Informations- und Buchungsplattform verstärkt genutzt werden.

Erhebliche Informationsdefizite bestehen besonders im Bereich der An- und Abreisemöglichkeiten. Im Alpenraum gehen Reisende grundsätzlich von einer schlechten Erreichbarkeit mit öffentlichen Verkehrsmitteln aus. Der Informationsbedarf betrifft hier vor allem die örtliche Multimodalität und die Vernetzung der verschiedenen Mobilitätsformen. Die Anforderungen der EU-Verordnung 2017/1926 der Kommission vom 31.05.2017 zur Bereitstellung EU-weiter multimodaler Reiseinformationsdienste wird diese Entwicklung ebenfalls stark vorantreiben (EU 2017).

Auch die Umweltleistungen und das Engagement verschiedener Anbieter zur Reduktion von Treibhausgasemissionen sollten besser dargestellt und auf den jeweiligen Internetseiten so zu finden sein, dass sie für die Entscheidungsfindung herangezogen werden können. Beispielhaft sei hier die lokale Energiegewinnung in Skigebieten durch die Seilbahnunternehmungen oder die energetische Nutzung natürlicher Heißwasserquellen in Thermen genannt. Bislang sind solche Umweltinformationen auf den Webseiten oft zwar vorhanden, aber vielfach schwer zu finden (Schmied 2012).

Eine sachgerechte Information und Kommunikation zum Klimawandel sind ein aufwendiger Prozess, der von den Betrieben und Destinationen sorgfältig geplant werden muss. Ansatzpunkte finden sich dazu für Österreich unter anderem in Prutsch et al. (2014). Die internationale Literatur zeigt aber auch, dass Information alleine nicht ausreicht (z. B. Stehr und

\footnotetext{
${ }^{4}$ Das bedeutet: die Zurechnung der externen Umwelteffekte auf den Verursacher.
} 


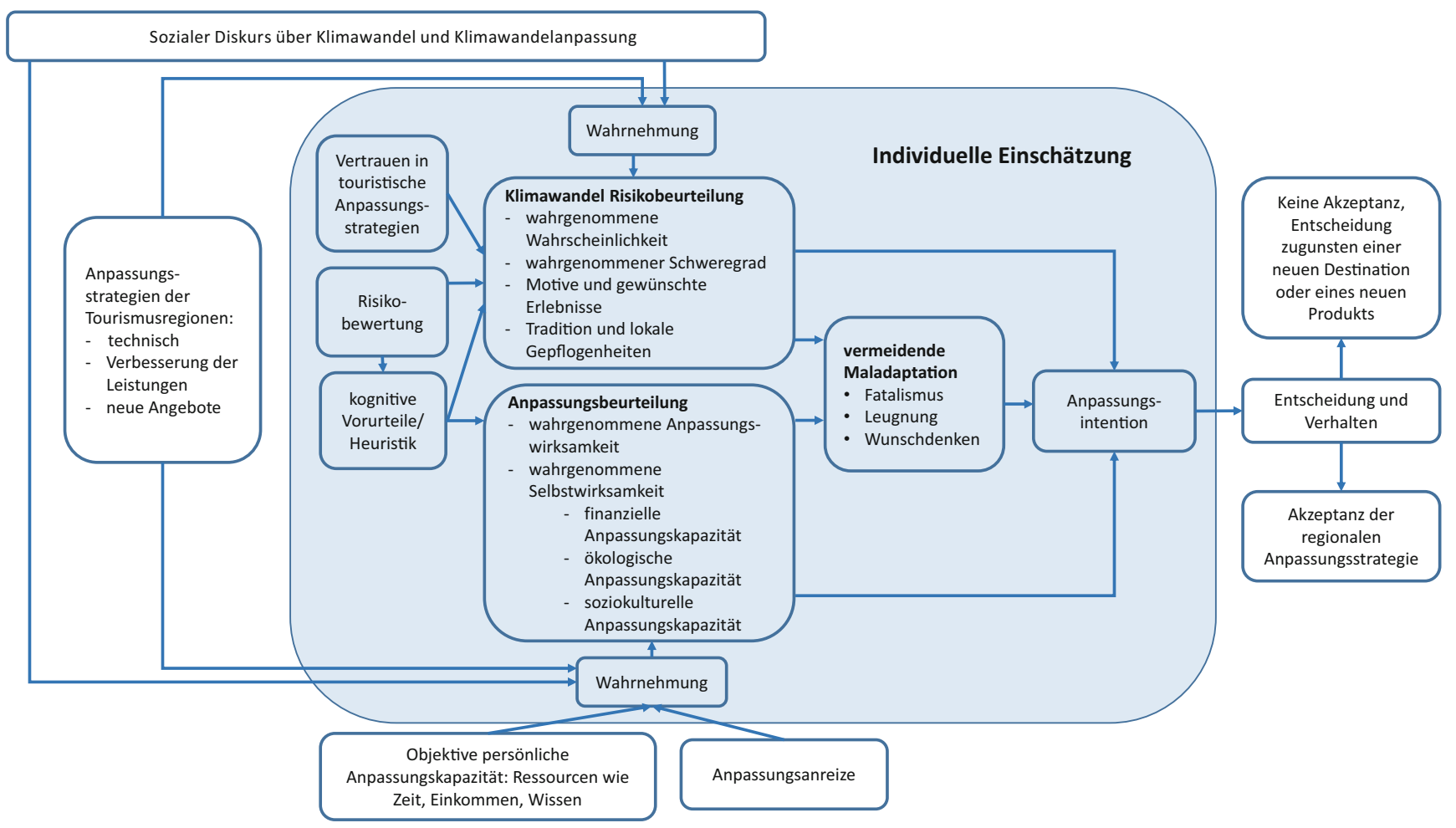

Abb. 13.1 Modell zur Anpassung von Touristen an den Klimawandel. (Verändert nach Pröbstl-Haider und Haider 2013; in Anlehnung an Grothmann und Patt 2005; Nachdruck aus Pröbstl-Haider und Haider 2013 mit Genehmigung von Emerald Publishing Limited)

von Storch 1995; Becken und Simmons 2008; Hunter und Shaw 2006; Sharpley 2006).

\section{Die Entscheidungen der Reisenden verstehen}

Die Klimawandelfolgenforschung in Europa beschäftigt sich seit rund 20 Jahren mit der Wahrnehmung, Bewertung und Abwägung möglicher Auswirkungen sowie dem Effekt auf die Anpassungsintention und das tatsächliche Verhalten (u. a. Stehr und von Storch 1995; Grothmann und Patt 2005; Grothmann et al. 2009; Pütz et al. 2011).

Das oben abgebildete Modell (Abb. 13.1) trägt dazu bei, die Wahrnehmungsprozesse und die verschiedenen Einflussfaktoren nachvollziehen zu können, und kann die Entwicklung von Strategien auf der Anbieterseite unterstützen. Dabei ist zwischen der internen Wahrnehmung und Bewertung, die zum Verhalten führen, und den externen Effekten durch Entwicklungen im gesellschaftlichen Umfeld oder in den Destinationen zu unterscheiden.

Extreme Klimabedingungen (z. B. die Dürre in Mitteleuropa 2018), die kritische Berichterstattung in den Medien sowie die Demonstrationen junger Menschen für weitreichendere politische Weichenstellungen unter Beachtung des Klimawandels (Fridays for Future) prägen die aktuelle gesellschaftliche Auseinandersetzung mit dem Thema. Auch der Tourismus, insbesondere Flugreisen, wird vermehrt kritisch diskutiert (vgl. Boeing 2019; DIE ZEIT 2019). Diese externen Diskurse können die interne Wahrnehmung möglicher
Risiken des Klimawandels beeinflussen (vgl. Einfluss auf die Erkenntnis in Abb. 13.1). Weiterhin spielt für diese Wahrnehmung auch das Engagement der Destination im Hinblick auf die Klimawandelanpassung eine wichtige Rolle. In diesem Zusammenhang ist etwa die Beschneiung von Skipisten oder die Bewässerung von Golfplätzen zu nennen, aber auch die Entwicklung neuer Angebote im Naturtourismus. Ob es zu einem veränderten Verhalten kommt, wird darüber hinaus davon beeinflusst, wie die Anpassungsmöglichkeit insgesamt beurteilt wird. Dabei spielen Fragen wie: „Kann ich mir ein Skigebiet mit umfangreicher Beschneiung leisten?" oder „Sind die von der Destination angebotenen Anreize (z. B. reduzierte Familienpreise) für mich so attraktiv, dass ich den Urlaubsort wechsle?", eine wichtige Rolle. Das Modell bezieht auch diese äußeren Einflüsse mit ein und beschreibt dann die individuelle Wahrnehmung im Hinblick auf die Beurteilung des Klimawandels und im Hinblick auf die Anpassung. So könnten dann, wenn eine Anpassung durch die Destination oder wichtige Einrichtungen vorliegt, die potenziellen Gäste selbst weniger eigenen Anpassungsbedarf erkennen oder aber ihre eigenen Anstrengungen ebenfalls erhöhen.

Die wichtigsten Komponenten des Modells sind die Klimawandelrisikobeurteilung (der Reisende bewertet die Wahrscheinlichkeit und den Schweregrad einer Beeinträchtigung für den geplanten Urlaub) und die Anpassungsbeurteilung (der Reisende bewertet die Wirksamkeit von Anpassungsmaßnahmen sowie den möglichen Aufwand solcher Maß- 
nahmen). Der Reisende könnte das Thema Schneesicherheit aus der medialen Diskussion aufgreifen und die Anpassungsstrategien im Hinblick auf eine technische Beschneiung zur Kenntnis nehmen. Zum Beispiel könnten die Reisenden bei der Entscheidung für einen Winterurlaub die Risiken im Zusammenhang mit der Schneesicherheit (unzureichende bzw. schlechte Schneelage) als gravierender einstufen als die vor Ort gegebenen Anpassungsstrategien durch die Beschneiung. Daher entscheidet sich der Reisende in diesem Beispiel für einen hoch gelegenen Wintersportort mit hoher natürlicher Schneesicherheit. Die hier dargestellten kognitiven Prozesse können als die wichtigsten Einflussfaktoren für die Verhaltensabsicht und das Verhalten gelten und helfen Entscheidungsprozesse nachzuvollziehen und zu modellieren (Grothmann und Patt 2005; Pröbstl-Haider und Haider 2013).

Der Tourismus unterscheidet sich von vielen anderen vom Klimawandel betroffenen Sektoren insofern, als die präferierte Anpassungsoption auch die Wahl einer anderen Destination mit ganz anderen Eigenschaften umfassen könnte (statt eines Skigebiets wird eine Flugreise in die Karibik gewählt). Diese Flexibilität beeinflusst auch die Risikobewertung erheblich und unterscheidet sie von Fallbeispielen aus anderen Formen der Landnutzung.

Das Modell berücksichtigt auch das Konzept der „Vermeidung von Fehlanpassungen" zwischen den Bewertungen und dem beabsichtigten Verhalten. Wenn zum Beispiel ein potenzieller Gast ganz besonders an einem bestimmten Wintersportort interessiert ist, könnte dieser Gast dann, wenn er eher „fatalistisch“ eingestellt ist (siehe Abb. 13.1), seine präferierte Skidestination trotz Schneemangels aufsuchen. Die starke Präferenz könnte eine (oder mehrere) negative Erfahrungen erfordern, bevor diese Person über Alternativen nachdenkt, wie zum Beispiel zukünftig eine Destination in schneesicherer Höhenlage zu wählen.

Das Erkennen entsprechender Systemzusammenhänge und Dynamiken ist die Voraussetzung, um weitere Strategien und Anpassungskonzepte entwickeln zu können. Hierzu zählen unter anderem saisonale Verschiebungen beziehungsweise Erweiterungen und Entwicklung nachhaltiger Produkte sowie die Wechselwirkungen von Anpassungsstrategien im Winter auf das Produkt im Sommer. In diesem Komplex sind vertiefende Forschungsarbeiten erforderlich.

\section{Die Entscheidungen der Reisenden beeinflussen}

Die Entscheidungen der Reisenden können, wie oben dargestellt, durch Informationen beeinflusst werden. Das bedarf jedoch einer differenzierten Informationsdarstellung sowie einer aktiven Auseinandersetzung des Gastes mit den Inhalten. Das kann sinnvoll und notwendig sein, könnte jedoch auch das Urlaubserlebnis negativ beeinflussen. Forschungsergebnisse von Dolnicar et al. (2017a, b) zeigen, dass die Bereitschaft der Touristen eher gering ist, an den wertvollsten Tagen des Jahres - ihren Ferien - bestimmte umwelt- freundliche oder energiesparende Maßnahmen zu ergreifen. So haben vergleichende Studien in Hotels dann nur geringe Auswirkungen gezeigt, wenn die Touristinnen und Touristen durch Flyer, Ankündigungen oder andere Mittel der Hotelinformation um ein entsprechendes umweltverträgliches Verhalten gebeten wurden. Rapp (2017) zeigt sogar, dass die Bereitschaft zur Wiederverwendung der Handtücher abnahm, wenn moralische Umweltargumente im Vergleich zu einer neutralen Kommunikation verwendet wurden. Auch andere Studien zeigen, dass selbst umweltbewusste und klimasensitive Touristinnen und Touristen beim Urlaub entgegen ihren Einstellungen handeln und durch Information und Kommunikation nur geringe Effekte erzielt werden können (Butcher 2003; Poon 2003; Simmons und Becken 2004; Hunter und Shaw 2006; Sharpley 2006; Becken und Simmons 2008).

Die einschlägige Literatur zeigt darüber hinaus eine signifikante Verschiebung in der Wahl der wissenschaftlichen Methoden, die zur Erforschung des Verbraucherverhaltens eingesetzt werden. Während jahrzehntelang die Theorie des geplanten Verhaltens (Ajzen 1985, 1991) als wichtiger Rahmen für die Abschätzung des Verbraucherverhaltens herangezogen wurde, wird sie heute nicht mehr als geeignet angesehen, um die Interventionen zu planen und zu gestalten, die effektive Verhaltensänderungen bewirken (Hardeman et al. 2002; Webb et al. 2010; Pröbstl-Haider und Haider 2013). Aktuelle Forschungen schlagen daher vor, sich auf neue Konzepte zu konzentrieren, die Forschungsergebnisse aus Psychologie, Ökonomie und Verbraucherverhaltensforschung zusammenführen, um Verhaltensänderungen bei den Gästen zu erreichen (für die verschiedenen Handlungsfelder bzw. Typen von Instrumenten, die zum Einsatz kommen können, siehe Abschn. 13.3.1-13.3.6). Aktuelle Forschungskonzepte zielen deshalb darauf ab, alternative Konzepte anzudenken, die über Einschränkungen, Umwelthinweise oder appellative Aufrufe hinausgehen, diese zu testen und umzusetzen. $\mathrm{Zu}$ diesen Ansätzen gehört unter anderem die Nudge-Theorie (Thaler und Sunstein 2008). Ein Nudge verändert das Verhalten der Menschen auf vorhersehbare Weise, ohne Möglichkeiten zu verbieten oder ihre wirtschaftlichen Anreize wesentlich zu ändern (Thaler und Sunstein 2008). Das bedeutet, dass touristische Produkte und Angebote so gestaltet und präsentiert werden, dass sie zu einer Verringerung der Treibhausgasemissionen führen, ohne das Urlaubserlebnis zu beeinflussen oder zu beeinträchtigen. ${ }^{5}$ Erste positive Nudging-Effekte wurden durch Änderung der Infrastruktur, Änderung der Standardoptionen sowie durch Erhöhung des Komforts und des Vergnügens erzielt (z. B. BacaMotes et al. 2013; Dolnicar et al. 2018; Juvan et al. 2018). Beispielhaft sei hier die Reduktion von Essensabfällen durch eine Verkleinerung der Teller oder durch Servieren von Speisen anstelle von Buffet-self-Service genannt. Solche Interventionen bieten, zusammen mit Gamification- und lifestyleorientierten

\footnotetext{
${ }^{5}$ Für Beispiele dazu siehe auch Abschn. 5.4.5.
} 
Angeboten, innovative Möglichkeiten für indirekte Verhaltensänderungen. Entsprechende Forschungsansätze, die Theorie und Praxis verbinden, stehen in Österreich noch aus.

Vor dem Hintergrund der eingangs erwähnten teilweise frustrierenden Ergebnisse bei dem Versuch, das Verhalten durch traditionelle Methoden der Information und Kommunikation zu beeinflussen, sehen wir daher in der NudgeTheorie einen vielversprechenden neuen Rahmen für eine Verbesserung des nachhaltigen Tourismusmanagements. Wir betrachten das Nudging nicht so sehr als Revolution, sondern als logische Weiterentwicklung einer sich wandelnden Governance-Landschaft (Baldwin et al. 2011; Levi-Faur 2011; Bradbury et al. 2013; Kosters und van der Heijden 2015).

Das bedeutet, dass Maßnahmen und touristische Angebote zu entwickeln sind, bei denen auch nichtklimabewusste Konsumentinnen und Konsumenten die Erwartungen an ihren Aufenthalt (Komfort, Erlebniskomponente etc.) erfüllt sehen, während sie (quasi unbemerkt) klimaschonend konsumieren. Um dieses Ziel zu erreichen, besteht erheblicher Forschungsbedarf.

Die Handlungsoptionen bezogen auf die Reisenden zielen darauf ab, einen „Paris Lifestyle“ zu erreichen:

- Beachtung der Klimaziele bei der Wahl des Urlaubsorts, des Betriebes und der Aktivitäten,

- Beachtung der Klimaziele bei der Wahl des Verkehrsmittels bzw. der Verkehrsmittel,

- Kompensation, insbesondere bei Flugreisen, und

- klimabewusste Entscheidungen.

Dies kann bezogen auf den Reisenden durch verschiedene Strategien aufseiten der Anbieter unterstützt werden:

- Qualität, die nicht darauf ausgerichtet ist, Abstriche bei den Kundinnen und Kunden zu verlangen,

- Anreize, die als Angebotserweiterung und Produktentwicklung empfunden werden,

- Nudges, die nicht spürbar sind,

- Erhalt bzw. Erweiterung des Erlebnisses als Prämisse und

- Beeinflussung der gesellschaftlichen Wahrnehmung bestimmter Urlaubsangebote.

\subsubsection{Anpassungs- und Vermeidungsmaßnahmen durch den Betrieb}

Neben den oben angesprochenen Änderungen des individuellen Verhaltens des einzelnen Reisenden bestehen zahlreiche Möglichkeiten, die Treibhausgasemissionen durch eine Veränderung der Anlagen, der betrieblichen Abläufe und der Anpassung bewährter Geschäftspraktiken zu reduzieren. In der Vergangenheit lag das Hauptaugenmerk auf der Angebotsseite, einschließlich der Verbesserung von Gebäuden und Dienstleistungen durch die Implementierung von Infrastruktur für erneuerbare Energien, energieeffizienten Anlagen und Isolierung (BMWFW et al. 2015) sowie der Unterstützung einzelner Betriebe durch Beratung, Zertifizierung und Förderung (Pröbstl und Müller 2012; APCC 2014; EIW 2014). Bei der Fortführung dieser Tradition gilt es, zukünftig bei den Förderungen die Klimafitness des zu fördernden Projektes jeweils noch mehr als bisher zu berücksichtigen bzw. Förderungen ggf. dahin gehend einzuschränken. Die Österreichische Tourismusbank hat bereits entsprechende Schritte gesetzt, die noch weiter ausgebaut werden könnten (klimaaktiv 2016). Im Hinblick auf die Zertifizierung und Auditierung gibt es im Tourismus Bereiche, in denen im Unterschied zu anderen europäischen Ländern Vorbilder und entsprechende Anwendungen fehlen. Dies gilt zum Beispiel für Golfanlagen, die in Spanien regelmäßig zertifiziert sind (Fürnweger 2016).

Ebenfalls weiterhin zu unterstützen sind alle Anstrengungen, die eine Zertifizierung von Betrieben fördern. Gerade im Energiesektor sind erhebliche Einsparpotenziale in der Vergangenheit nachgewiesen (Reschl 2019). Durch Förderungen oder andere Anreize sollte die Entscheidung zu einer regelmäßigen Zertifizierung leichter gemacht werden. Viele Betriebe würden sich in diesem Zusammenhang Erleichterungen bei der Genehmigung von Anpassungsmaßnahmen wünschen (Pröbstl-Haider et al. 2018).

Darüber hinaus wird heute von den Betrieben auch erwartet, dass sie das Angebot an klimaverträglichen Produkten und Dienstleistungen schrittweise vergrößern. So wird vom Kunden vielfach bereits gewünscht, dass neben der klimaschonenden Übernachtung auch Anreise, Mobilität vor Ort und angebotene Aktivitäten entsprechend mitgebucht werden können. Diese Angebotsentwicklung geht oft über die Kernkompetenzen des einzelnen Betriebes hinaus und erfordert Kooperationen auf Destinationsebene (vgl. Abschn. 13.2.3).

Erschwerend kommt hinzu, dass aus betrieblicher Sicht häufig bei Anpassungsmaßnahmen Ressourceneffizienz und Gästekomfort gegeneinander abgewogen werden müssen. Dies trifft zum Beispiel die Temperatur der Räume, die Wärme des Pools, das Entfernen der Minibar u. v. a. mehr. Ein weiteres häufig genanntes Hindernis ist die Sorge der Betriebe, dass Einsparungsmaßnahmen zulasten der Dienstleistungen beziehungsweise der Arbeitsbedingungen ihrer Mitarbeiter gehen könnten (Becken 2013).

Wege zu einem klimaverträglichen touristischen Produkt können von einzelnen Betrieben meist nicht alleine umgesetzt werden. Partnerschaften und Kooperationen auf Destinationsebene sind vielfach bei der Produktentwicklung zwingend notwendig. 
Tab. 13.1 Potenzielle Effekte neuer klimaschonender Produkte mit geringeren Treibhausgasemissionen. (Verändert nach Holloway und Robinson 1995, S. 82; Zeithaml und Bitner 1996, S. 201; Komppula 2001, S. 9)

\begin{tabular}{|c|c|c|}
\hline Angebote & $\begin{array}{l}\text { Existierende } \\
\text { Märkte/bestehende } \\
\text { Gästesegmente }\end{array}$ & $\begin{array}{l}\text { Neue Märkte/neue } \\
\text { Gästesegmente }\end{array}$ \\
\hline $\begin{array}{l}\text { Bestehende } \\
\text { Produkte } \\
\text { mit Re- } \\
\text { duktion von } \\
\text { Treibhaus- } \\
\text { gasemis- } \\
\text { sionen }\end{array}$ & $\begin{array}{l}\text { Marktdurch- } \\
\text { dringung } \\
\text { Modifikationen } \\
\text { bestehender } \\
\text { Produkte für den } \\
\text { bestehenden Markt } \\
\text { und Reduktion von } \\
\text { Treibhausgasemis- } \\
\text { sionen }\end{array}$ & $\begin{array}{l}\text { Marktentwicklung } \\
\text { Neupositionierung eines } \\
\text { bestehenden Produktes, } \\
\text { um einen neuen Markt } \\
\text { und neue Kunden- } \\
\text { segmente mit Interesse } \\
\text { an klimaschonenden } \\
\text { Produkten anzusprechen }\end{array}$ \\
\hline $\begin{array}{l}\text { Neue Pro- } \\
\text { dukte zur } \\
\text { Reduktion } \\
\text { von Treib- } \\
\text { hausgas- } \\
\text { emissionen }\end{array}$ & $\begin{array}{l}\text { Neuentwicklung } \\
\text { Einführung von } \\
\text { neuen Produkten } \\
\text { zur Einsparung von } \\
\text { Treibhausgasemis- } \\
\text { sionen in einen } \\
\text { bestehenden Markt }\end{array}$ & $\begin{array}{l}\text { Diversifizierung } \\
\text { Einführung eines neuen } \\
\text { klimaschonenden } \\
\text { Produktes und Er- } \\
\text { schließung eines neuen } \\
\text { Marktes bzw. neuer } \\
\text { Kundensegmente }\end{array}$ \\
\hline
\end{tabular}

Neue Produktentwicklungen können auch dazu dienen, neue Gästesegmente anzusprechen, wie Tab. 13.1 zeigt. Bonadonna et al. (2017) erwarten in diesem Zusammenhang eine Attraktivitätsverbesserung, die vor allem bei der Gruppe der sogenannten Millennials (Personen, die zwischen 1980 und 1995 geboren sind) wirksam ist, weil für dieses Segment das Thema Nachhaltigkeit bereits einen höheren Stellenwert besitzt. Die Autoren untersuchten dies im Zusammenhang mit dem Bergtourismus in Italien.

Um die betrieblichen Anpassungen zu erreichen, können zusammenfassend folgende Ansätze dienlich sein.

Die Handlungsoptionen bezogen auf die Betriebe zielen darauf ab, die Treibhausgasemissionen zu reduzieren und dem Gast einen Urlaub im Sinne des "Paris Lifestyle" zu ermöglichen:

- Verbesserungen auf der Angebotsseite durch Verbesserung der Anlagen, der betrieblichen Abläufe und Anpassung von Geschäftspraktiken;

- Ausbau von Beratung, Zertifizierung und Förderung bei allen Anbietern;

- schrittweiser Ausbau klimaverträglicher Produkte und Dienstleistungen einschließlich Mobilität vor Ort sowie der An- und Abreise, ggf. mit weiteren Partnern;

- Kommunikation von Abwägungsentscheidungen zugunsten von Ressourceneffizienz;

- Nutzung von Anpassungsmaßnahmen zur Neupositionierung, um einen neuen Markt und Kundensegmente mit Interesse an klimaschonenden Produkten anzusprechen.

\subsubsection{Anpassungs- und Vermeidungsmaßnahmen durch die regionale Destination}

Folgt man den gesetzlichen Vorgaben, liegen die Innovation und Weiterentwicklung der touristischen Angebote in Österreich eindeutig bei den Tourismusverbänden. Dementsprechend haben die regionalen Tourismusverbände als Destinationen die Aufgabe, das Angebot von klimaverträglichen Produkten und Dienstleistungen zu vergrößern, um den Tourismus in ihrer Region auf einen klimaverträglichen Kurs zu bringen. Pröbstl (2011) zeigt, dass dies gerade bei Verbänden mit heterogenen Verhältnissen, wie etwa im Ennstal, kein einfacher und vielfach konfliktreicher Weg ist, weil die regionalen Einheiten nicht immer den touristischen Netzwerken entsprechen. So organisieren sich zum Beispiel die großen Skigebiete im Westen des steirischen Ennstals über die Landesgrenzen hinweg mit Salzburger Betrieben zur Skiwelt Amadé. Ein anderes Beispiel für Zusammenschlüsse, die unabhängig von den touristischen Strukturen sind, stellen etwa die Klima- und Energiemodellregionen (KEM) oder die Klimawandelanpassungsregionen (KLAR!) dar, welche zum Motor für Anpassungs- und Vermeidungsmaßnahmen werden können (Salak 2016).

Nachhaltige Anpassungsstrategien, das zeigten auch die Stakeholderworkshops ${ }^{6}$, beziehen auch die Belange der einheimischen Bevölkerung und deren Lebensqualität mit ein:

Dabei sollte vermieden werden, dass Erholungseinrichtungen für den Tourismus bzw. deren ressourcenschonende Anpassung von der Region, den Bürgerinnen und Bürgern finanziert oder mitfinanziert werden, diese die entsprechenden Einrichtungen und Angebote jedoch nicht (oder nicht einmal verbilligt) nutzen können. Ähnliches gilt für Mobilitätsangebote der Region, bei denen die Gäste umsonst klimaneutral an- und abreisen können, die von der lokalen Bevölkerung jedoch nicht kostenfrei benutzt werden können. Eine integrierte Sichtweise ist daher erforderlich. Diese sollte auch regionalökonomische Belange (Wertschöpfung in der Region), soziale und ökologische Aspekte berücksichtigen. Diese Abwägung erfordert in der Regel die aktive Beteiligung der Kommunen und teilweise auch der Regionen.

Um die regionalen Anpassungen zu erreichen, können zusammenfassend folgende Ansätze sinnvoll sein:

\footnotetext{
${ }^{6}$ Im Rahmen der Erstellung des Berichtes wurden verschiedene Stakeholderworkshops durchgeführt.
} 
Die Handlungsoptionen bezogen auf die Regionen zielen darauf ab, Ansätze zur Einsparung von Treibhausgasemissionen zu bündeln, Synergieeffekte zu nutzen und branchenübergreifende Netzwerke zu bilden:

- Die Tourismusverbände müssen den Klimaschutz als weiteres Handlungsfeld erkennen und bearbeiten.

- Regionale Anpassungen auf Destinationsebene erfordern die Zusammenarbeit und Mitwirkung unterschiedlicher Partner.

- Die Entwicklung geeigneter Anpassungsstrategien sollte auch die Lebensqualität der einheimischen Bevölkerung miteinbeziehen und ökologische, ökonomische und soziale Aspekte beachten.

- Den Regionen kommt im Hinblick auf die Kommunikation eine zusammenführende und ordnende Funktion zu. Regional können auch Schwerpunkte, wie etwa eine vegane Skidestination, besser nach außen vermittelt werden.

- Kommunen und andere Organisationsstrukturen sollten bei regionalen oder destinationsübergreifenden Initiativen intensiv miteingebunden werden.

\subsubsection{Anpassungs- und Vermeidungsmaßnahmen durch die Destination Österreich}

Flankierende Maßnahmen auf nationaler und europäischer Ebene sind über die Maßnahmen auf der Destinationsebene hinaus unbedingt erforderlich. Dies gilt insbesondere im Bereich Verkehr, wo durch entsprechende Besteuerung und die Bereitstellung von öffentlichen Verkehrsmitteln wesentliche Rahmenbedingungen für die Anpassung einerseits und neue Produktentwicklung andererseits geschaffen werden können. Die persönlichen Abwägungsentscheidungen der Reisenden werden von der Entwicklung dieses Angebotes wesentlich abhängen.

Auf nationaler Ebene werden vor allem Marketing und Außendarstellung der Urlaubsregion Österreich wesentlich beeinflusst. Der Klimawandel und entsprechende Anpassungsstrategien lassen sich hier bislang noch nicht erkennen. Auch in diesen Bereichen sind die Darstellungen und die Vermarktung nach außen in Einklang mit der oben beschriebenen Vision kritisch zu überprüfen und anzupassen.

Auf nationaler Ebene können darüber hinaus weitere hoheitliche Lenkungsmaßnahmen in Betracht gezogen werden. Beispielhaft sind hier eine ökologische Steuerreform mit dem mittelfristigen Ziel einer vollständigen Internalisierung externer Kosten sowie eine nationale Förderpolitik genannt, die die Transformation hin zu einer nachhaltigen, klimaschonenden Entwicklung finanziell unterstützt und für Betriebe bzw. Regionen die Umstellung erleichtert bzw. wirtschaftlich möglich macht (Mundt 2011). In diesem Zusammenhang ist es erforderlich, dass das Wirtschaftssystem, in das der Tourismus eingebunden ist, insgesamt auf einen klimaverträglichen Kurs gebracht wird.

Um die nationalen Anstrengungen zur Vermeidung und Anpassungen zu fördern, können zusammenfassend folgende Ansätze erforderlich werden:

Die nationalen Handlungsoptionen zielen darauf ab, die Rahmenbedingungen durch Wirtschaftsförderung, Besteuerung oder öffentliche Angebote (z. B.

Mobilität) so zu beeinflussen, dass Anreize zur Einsparung von Treibhausgasemissionen entstehen und die Eigeninitiativen auf regionaler, lokaler und persönlicher Ebene gefördert werden:

- Lenkungs- und Steuerungsmaßnahmen könnten auf eine Internalisierung der externen Kosten beim Reisen hinwirken.

- Die nationale Transformation hin zu einer nachhaltigen, klimaschonenden Entwicklung sollte auf allen Ebenen gefördert werden.

- Das nationale Außenmarketing sollte gezielt an die Vision des „Paris Lifestyle“ angepasst werden. Dazu gehört auch eine bevorzugte Bewerbung Österreichs in näher liegenden Räumen und weniger im Fernreisemarkt.

- Einführung von Kennwerten zur Erfassung der Emissionsbelastung bzw. Nachhaltigkeit im Bereich des Tourismus.

\subsection{Spezifische Strategien und konkrete Handlungsoptionen}

Der vorliegende Bericht kann nicht die Vielzahl der Möglichkeiten abbilden, die zur Anpassung an den Klimawandel denkbar sind. Der Abschn. 1.2 hat bereits aufgezeigt, auf welchen Ebenen und in welcher Weise wichtige Impulse gesetzt werden können. Zum Abschluss des Berichts widmen wir uns daher spezifischen Strategien und daraus ableitbaren Handlungsoptionen, die sich speziell an die Politik und Personen auf der Entscheidungsebene wenden.

Aus wissenschaftlicher Sicht lassen sich sechs Felder von Handlungsoptionen unterscheiden (vgl. Mostegl 2020 und Abb. 13.2), von denen jedes für sich seine Berechtigung und spezielle Anwendungsgebiete hat. Die methodischen Herangehensweisen unterscheiden sich jedoch in ihrer Wirkungsweise und Effizienz. Nachstehend sind diese Handlungsfelder 


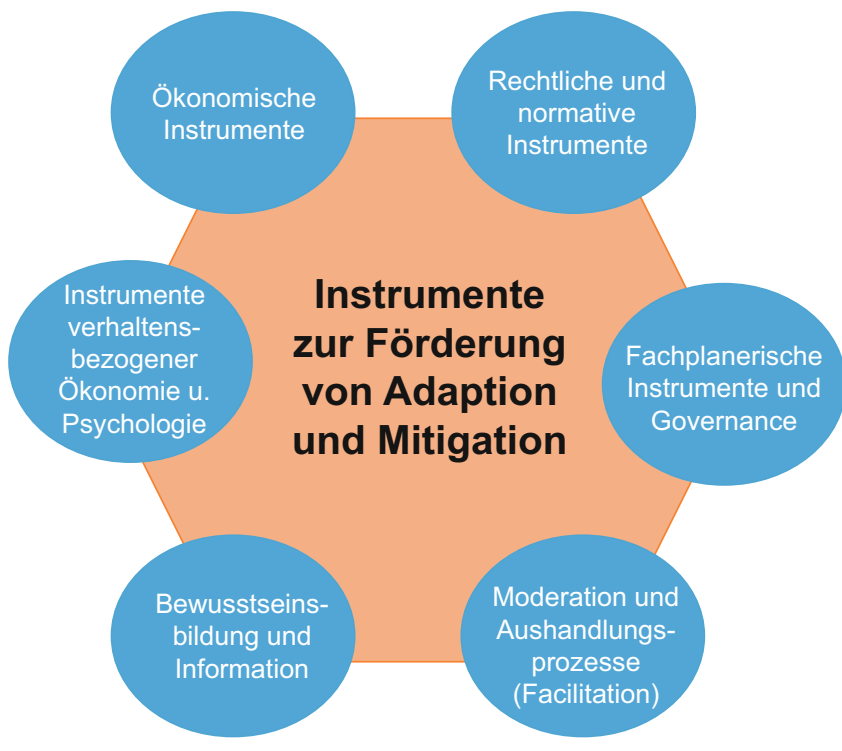

Abb. 13.2 Instrumente zur Förderung von Adaptions- und Mitigationsprozessen im Zusammenhang mit dem Klimawandel. (Verändert nach Mosteg1 2020; Grafik: Ulrike Pröbstl-Haider)

und dafür beispielhafte Optionen beschrieben. Die Beschreibungen sollen weiterhin verdeutlichen, welche Überlegungen sich daraus für die Branche ergeben und mit welchen Herausforderungen im Einzelfall zu rechnen ist. Welche Fragestellungen sich dabei in der Umsetzung für Forschung und Praxis ergeben, wird jeweils an mehreren Fallbeispielen illustriert. Für die Formulierung der Fragen wurden jeweils aktuelle Trends und Entwicklungen herangezogen und ,weitergedacht". Kernfragen werden dann durch weiterführende Fragestellungen konkretisiert und veranschaulicht. Die Fragenblöcke sind - um die Diskussion anzuregen - jeweils farblich hervorgehoben.

\subsubsection{Rechtliche und normative Instrumente}

Rechtliche und normative Instrumente setzen auf rechtlich verbindliche Lösungen, um die gewünschten Ziele umzusetzen. Die jeweiligen Regelungen sind verbindlich und ihre Einhaltung wird daher von der Exekutive und der Judikative überwacht und durchgesetzt. Zu den rechtlichen und normativen Steuerungsmöglichkeiten gehören insbesondere Gesetze, Verordnungen oder Richtlinien, die Gebote, Verbote, Auflagen und Normen enthalten können. Diese Instrumente werden in der Regel eingeführt, um für eine Vielzahl von Fällen eine gleichberechtigte Regulierungsgrundlage zu schaffen und gleichzeitig die Notwendigkeit zu umgehen, Einzelvorschriften zu erlassen und wiederholt grundlegende Wertentscheidungen treffen zu müssen (Mostegl 2020). Sie sind vor allem wichtig für eine langfristige Planung und sollten daher eine hohe Verlässlichkeit aufweisen. Gerade vor diesem Hintergrund ist es eine große Herausforderung, die Auswirkungen und Implikationen - für die Tourismuswirtschaft einerseits sowie für die Gesellschaft andererseits - jeweils frühzeitig abzuschätzen.

Die Europäische Union hat bereits verbindliche Vorgaben für die Energiewende gemacht. Jeder Mitgliedstaat ist verpflichtet diese entsprechend einzuhalten und in nationales Recht zu überführen. Bislang wurde diese Verpflichtung aus der Sicht der Experten zu wenig beachtet, ebenso wie die Tatsache, dass eine Nichterfüllung in der Regel Sanktionen nach sich ziehen wird (VCÖ 2019).

Die Wirkung von normativen Instrumenten kann man anhand der folgenden beispielhaften Fragestellungen diskutieren (Abb. 13.3).

In der Vergangenheit hat die Europäische Union keine Zweifel daran gelassen, dass sie die von ihr vorgegebenen Normen um- und durchsetzt. Strafzahlungen gehören regelmäßig dazu. Wird das Einsparungsziel an Treibhausgasemissionen verfehlt, kommt ein innereuropäischer Zertifikatshandel zum Tragen. Die geschätzten Kosten dafür liegen nur für den Verkehrsbereich im einstelligen Milliardenbereich (VCÖ 2019). Wie diese Zahlungen dann national umgelegt werden, nach Sektoren (Deutschland diskutierte im Herbst 2019 eine Sektorenlösung bezogen auf die Verantwortung von Ministerien) oder nach Bundesländern (wurde im Zusammenhang mit der Umsetzung der FFH-Richtlinie in Deutschland überlegt), ist Sache des jeweiligen Mitgliedstaates.

Die touristische Branche könnte die Entscheidungsgrundlagen wesentlich verbessern, indem ein Regelwerk zur Erfassung und Bilanzierung der ihr zuzuordnenden Treibhausgase aufgestellt wird, um Leistungen dokumentieren zu können, aber auch um ggf. sektorale Anlastungen zurückweisen zu können.

Weiterhin sollten die Effekte aus europäischen und internationalen Verpflichtungen, wie aus dem Abkommen von Paris, so detailliert wie möglich in die jeweilige Regierungsplanung aufgenommen werden, sodass eine Verantwortlichkeit auf die zuständigen Ressorts übertragen werden kann. Eine branchenoder sektorenbezogene Eigenverantwortung könnte aus dieser Bilanzierung, Diskussion und Zuordnung ebenfalls entstehen.

Normative Instrumente können aber auch gezielt Anreize für Anpassungen schaffen, um in der täglichen Praxis „,besser“ zu werden. Ein Beispiel dafür sind die Auflagen für klimaschonende Veranstaltungen. Im Rahmen der Erarbeitung des vorliegenden Berichts entstand der Eindruck, dass die Anforderungen an die Klimaverträglichkeit von Veranstaltungen inzwischen zu niedrig angesetzt sind. Hier könnten normative Vorgaben einen wichtigen Anreiz bieten, da viele Veranstalter gerne mit einem ,grünen Event“" werben wollen. Eine Herausforderung stellt allerdings die Frage dar, wie weitreichend die Vorgaben sein sollen und welche Auswirkungen bezogen auf die Austragungsorte (insbesondere periphere Lagen) und die dortige Wertschöpfung entstehen.

Normativ könnten auch touristische Anbieter verpflichtet werden, den jeweiligen Beitrag der angebotenen Reise, Un- 
Abb. 13.3 Beispielhafte Fragestellungen bei Nichterreichung der Einsparungsziele
Was passiert, wenn Österreich die Vorgaben der EU zur Senkung der Treibhausgasemissionen nicht erreicht? Momentan liegt die Zielvorgabe der Österreichischen Klima- und Energiestrategie (BMNT \& BMVIT, 2018) bei einer Reduktion um $36 \%$ im Vergleich zum Jahr 2005, auf europäischer Ebene wird hingegen bis 2030 eine Emissionsreduktion um mindestens $40 \%$ gegenüber dem Stand von 1990 verlangt.

$\rightarrow$ Wer würde dabei zur Rechenschaft gezogen werden?

$\rightarrow$ Wie hoch sind die finanziellen Mittel, die bei Nicht-Erfüllung oder die kurzfristig zur Erfüllung bereitgestellt werden müssen und wie wirkt sich dieser Eingriff in den Haushalt u. a. gesellschaftlich und wirtschaftlich aus?

$\rightarrow$ Wie würde sich die Nichterfüllung auf das Image Österreichs als nachhaltige Destination auswirken? terkunft, Tagesskipässe, Thermeneintritte usw., zu den Treibhausgasemissionen kenntlich zu machen, wie dies bereits heute einzelne Leitbetriebe der Seilbahnwirtschaft oder Anbieter von Busreisen tun. Insbesondere Buchungsplattformen könnten in diesem Zusammenhang nicht nur Preisvergleiche, sondern auch die Auswirkungen auf die Treibhausgasemissionen kommunizieren und einander gegenüberstellen müssen. Geeignete Berechnungsgrundlagen liegen dafür vor (u. a. ökologischer Fußabdruck; z. B. TU Graz o.J.).

Die Beispiele zeigen, dass die Effekte normativer Festlegungen jeweils mitgedacht werden müssen. Dies erfordert vielfach auch die vorausschauende Anwendung von anderen, nachstehend vorgestellten Instrumenten, insbesondere der Verhaltensökonomik oder der Aushandlung und Moderation.

\subsection{2 Ökonomische Steuerungsinstrumente}

Wirtschaftliche Instrumente lassen sich in finanzielle Unterstützung und Finanzierung, Steuern und Abgaben (Erhöhung und Befreiung), Entschädigungsregeln und handelbare Umweltlizenzen unterteilen (Schenker et al. 2014; Loer 2015; Thorun et al. 2017; Vetter et al. 2017). Derzeit zählen die Entwicklung und Umsetzung wirtschaftlicher Anreize zu den wichtigsten Instrumenten der Politikentwicklung. Entsprechende Ansätze streben entweder durch positive oder negative finanzielle Anreize eine Verhaltensänderung an. Positive Anreize, wie zum Beispiel Förderungen für energiebewusstes Bauen, können eine hohe finanzielle Belastung für den Staat darstellen, insbesondere dann, wenn das Instrument stark nachgefragt wird.

Negative finanzielle Anreize zielen darauf ab, Verhalten durch die Einführung von Sanktionen zu beeinflussen. So wird von vielen Seiten im Zusammenhang mit dem Klimawandel die Besteuerung von Kerosin gefordert, damit über die steigenden Kosten der Anreiz zu fliegen sinkt. Trotz der Steuereinnahmen ist der entstehende Verwaltungsaufwand bei Einführung nicht wesentlich geringer als bei der Implementierung anderer Regulierungsinstrumente. Weiterhin ist aus der Sicht der politischen Parteien eine Besteuerung immer auch ein Instrument, das - zumindest in Teilen der Bevölkerung - zu Ablehnung und Kritik führt beziehungsweise führen kann. Darüber hinaus weisen Biesbroek et al. (2013) und Schenker et al. (2014) darauf hin, dass wirtschaftliche Steuerungsinstrumente dieser Art nicht die Einstellungen der Betroffenen beeinflussen und daher oftmals keine nachhaltige Entwicklung anstoßen oder anstoßen können. In diesem Fall würde der Reisende zwar versuchen, Steuern zu vermeiden, nicht aber für den Klimaschutz eintreten.

Dennoch sind Steuern, trotz der geringen Akzeptanz in der Öffentlichkeit, die gängigsten politischen Vorgehensweisen, wenn es darum geht, unerwünschtes Verhalten zu beeinflussen (Buckley und Llerena 2018). Die Nutzung von finanziellen Anreizen über Steuern unterliegt zudem der Schwierigkeit, dass ihre Wirkung und Betroffenheit in der Bevölkerung in der Regel ungleich verteilt sind. Weiterhin stellt sich die Frage, ob die Förderungen bei ungleichen Verhältnissen auch dort ankommen, wofür sie gedacht sind. Aus touristischer Sicht gilt dies insbesondere für die ungleichen Verhältnisse zwischen städtischen und ländlichen Räumen. Entsprechende Initiativen erfordern daher eine intensive Vorbereitung und Absicherung. In diese Vorbereitung sind auch Folgeabschätzungen miteinzubeziehen (Abb. 13.4).

Die Auseinandersetzung mit der Einflussnahme auf weit entfernte Reisemärkte, wie etwa dem südostasiatischen Fernreisemarkt, und die damit verbundene Diskussion um entsprechende Werbemaßnahmen unterstreichen, wie wichtig eine Vorabschätzung möglicher Wirkungen ist. Die Diskussion mit Stakeholdern im Rahmen der Berichterstellung zeigte, dass für touristische Betriebe im Bundesland Salzburg der Fernreisemarkt wirtschaftlich wichtig ist, während die Bundesländer Niederösterreich und Steiermark eher eine Konzentration auf europäische Märkte mit länger bleibenden und zahlungskräftigen Gästen bevorzugen würden.

In diesem Zusammenhang muss auch diskutiert werden, ob beständig weiterwachsende Märkte das Ziel des österreichischen Tourismus sein sollen. Könnte nicht die Zielsetzung in Verbindung mit der Klimawandelanpassung darin bestehen, die Länge des jeweiligen Aufenthalts zu erhöhen, um nicht mehr auf eine permanent wachsende Zahl an Gästen mit 
Abb. 13.4 Beispiel für Fragestellungen zu den Effekten einer Besteuerung von Kerosin auf die inländische und ausländische Nachfrage und entsprechender Handlungsbedarf
Abb. 13.5 Beispiel für Fragestellungen zur Elektromobilität
Was wäre, wenn eine $\mathrm{CO}_{2}$-Steuer auf Kerosin eingeführt werden würde und dadurch die Hälfte $(50 \%)$ aller österreichischen Flugreisenden, die sonst im Ausland Urlaub machen, nunmehr ein touristisches Angebot im Inland nutzen wollen? Damit würden rund zwei Millionen Flugreisen entfallen und, bei einer durchschnittlichen Nächtigungsdauer von 3,3 Nächten, der Bedarf für rund 6,6 Mio. zusätzliche Nächtigungen in Österreich ( $+4,4 \%)$ entstehen.

$\rightarrow$ Sind diese zusätzlichen Übernachtungsgäste in der entsprechenden Saison unterzubringen?

$\rightarrow$ Welche Destinationen könnten von diesem Anstieg am stärksten profitieren und sollten diese vorab gezielt beworben werden, um die Entwicklung zu steuern?

$\rightarrow$ Wie können Nahmärkte im Allgemeinen stärker angesprochen werden und wie kann dem Trend zu einer kurzen Aufenthaltsdauer entgegengewirkt werden?

Eine Besteuerung von Kerosin würde sich aber auch auf den Fernreisemarkt auswirken und die Struktur der Gäste beeinflussen, die Österreich besuchen. Bislang wird erwartet, dass die Ankünfte aus Schwellenländern, vor allem aus Asien, weiter rasant ansteigen (Buckley et al., 2015).

$\rightarrow$ Könnte man diesen Trend, der die Tourismusmärkte in den nächsten 30 Jahren bestimmen soll, durch höhere Kerosinpreise ausreichend stark beeinflussen?

$\rightarrow$ Wie würde sich eine Zunahme von Fernreisenden unter den Gästen auf die Treibhausgasbilanz des österreichischen Tourismus auswirken? Wäre es damit immer noch möglich, die national angestrebten Ziele zur Emissionsreduktion zu erreichen?

$\rightarrow$ Ist es sinnvoll, Werbemaßnahmen zur Förderungen des Fernreisetourismus zu limitieren und stattdessen verstärkt auf Nahmärkte zu setzen?

Was wäre, wenn $20 \%$ der deutschen und niederländischen Urlauberinnen und Urlauber mit einem elektrischen Auto anreisen würden, wie es die Marktentwicklung vorsieht, die von einem Anteil von $15 \%$ bis $25 \%$ der Neuzulassungen bei ca. 100 zur Auswahl stehenden Modellen zukünftig ausgeht (NPE, 2018)?

$\rightarrow$ Beeinflusst die Elektromobilität die Anreiseform und Destinationswahl der nationalen, europäischen und internationalen Gäste?

$\rightarrow$ Wäre die notwendige Infrastruktur in den Destinationen vorhanden (E-Ladestationen)?

$\rightarrow$ Führt diese Entwicklung im ländlichen Raum zu Nachrüstungs- und Investitionsbedarf bei den Betrieben?

$\rightarrow$ Würden Rebound-Effekte durch erhöhte Fahrleistungen mit den E-Pkws entstehen und die Bereitschaft zur Nutzung öffentlicher Verkehrsmittel, wie Busse und Bahnen, sinken? immer kürzeren Aufenthaltszeiten setzen zu müssen. Es stellt sich damit indirekt auch die Frage, ob - wirtschaftlich gesehen - anhaltendes Wachstum (Becken 2016) oder ein Wachstumsparadigma für den Tourismus zwingend notwendig ist. Dies mag für weltweite Entwicklungen vielleicht zutreffen, bei einer Betrachtung spezifischer Regionen in Österreich erscheinen jedoch auch andere Lösungen denkbar, die auf stabile Strukturen abzielen und an einer Maximierung der Wertschöpfung ausgerichtet sind.

Auch bezogen auf die Elektromobilität werden Förderungen oder steuervergünstigende Lösungen nicht nur in Österreich, sondern vor allen in wichtigen Herkunftsmärkten österreichischer Gäste diskutiert. Daher ist es wichtig, sich mit den möglichen Effekten dieser Steuerungen auseinanderzusetzen (Abb. 13.5).

Derzeit wäre Österreich wohl nicht in der Lage, die erforderliche Versorgung mit Ladestationen für die E-Mo- bilität in den gewünschten Destinationen bereitzustellen. Daher könnte eine wichtige Handlungsoption darin bestehen - nachdem die Ausrichtung auf die Elektromobilität bei den großen Herstellern gefallen ist -, durch eine gezielte Förderung die Versorgungssicherheit auch abseits der Städte und Autobahnen herzustellen. Eine Befragung in Zusammenarbeit mit der Hoteliervereinigung (PröbstlHaider 2014a) zeigt, dass ein Interesse an einer flächendeckenden Versorgung besteht und viele Betriebe daran mitwirken würden. Immerhin $29 \%$ wünschen sich in $\mathrm{Zu}$ kunft eigene Ladestationen zu ihrem Hotelbetrieb, weitere $27 \%$ würden bei vorhandenen Förderungen entsprechende Anlagen im eigenen Betrieb errichten und $44 \%$ erwarten eine allgemeine Versorgung durch Dritte (Stadt, Gemeinde, Stromanbieter o. Ä.).

Neben der Einflussnahme durch Steuern gehören auch Anreize durch Förderungen zu besonders einflussreichen öko- 
Abb. 13.6 Die Befragung von Hotelbetrieben $2014 \mathrm{zu}$ den geplanten Heizungsanlagen $(n=$ 164) zeigt ein hohes Interesse an erneuerbaren Energieträgern. (Pröbstl-Haider 2014a)
Abb. 13.7 Die Planung von Maßnahmen durch die befragten Hotelbetriebe $(n=164)$ zur Energieeinsparung betrifft neben Energieeinsparung auch die Gewinnung erneuerbarer Energie sowie die Mobilität und den Bereich der Ernährung. (PröbstlHaider 2014a)

\section{Welche der folgenden Heizungsarten verwenden Sie derzeit bzw. welche Änderungen sind zukünftig geplant?}

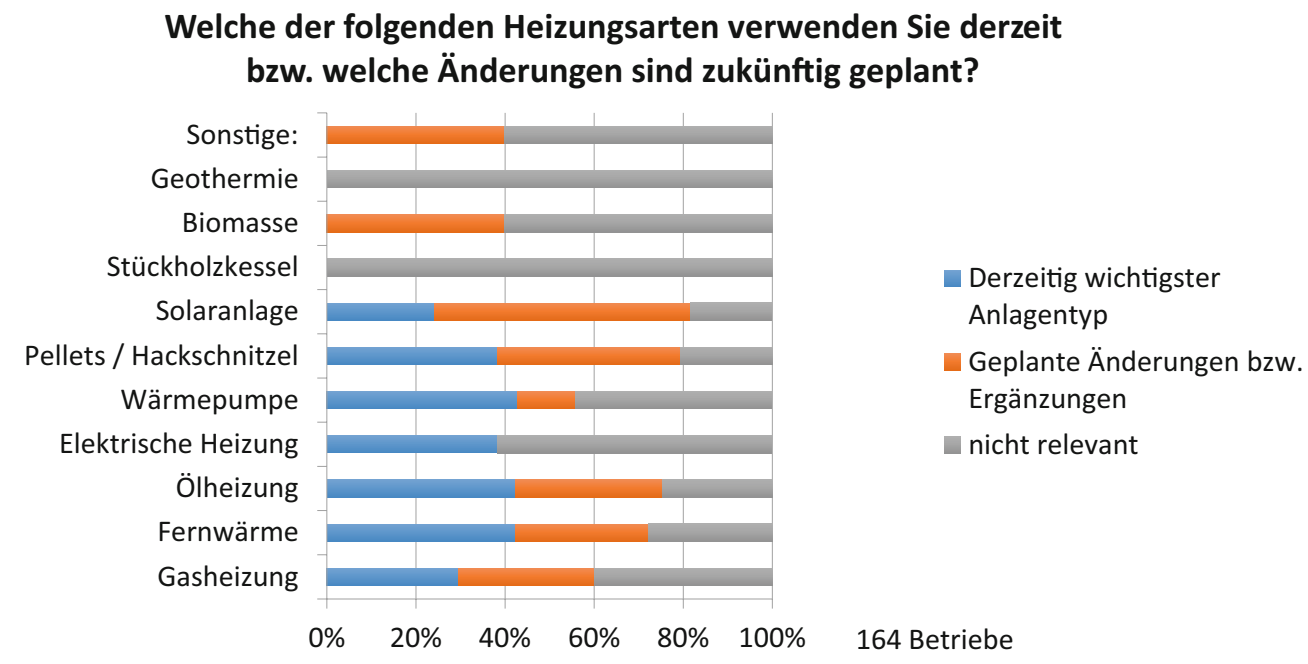
Welche der folgenden Maßnahmen zur Einsparung von Energie haben Sie bereits umgesetzt und welche Maßnahmen sind geplant?

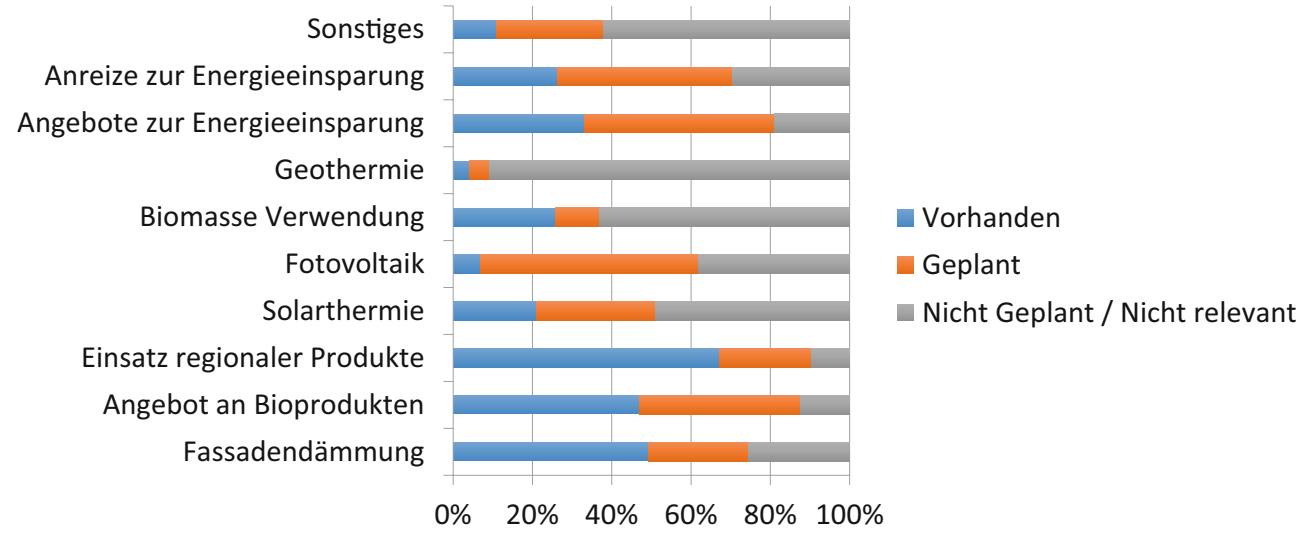

nomischen Instrumenten. In diesem Zusammenhang stellte die Europäische Umweltagentur (EEA 2014) fest, dass die projektbasierte, finanzielle Unterstützung sich als der wichtigste Finanzierungsmechanismus für die Umsetzung von Anpassungsmaßnahmen herausgestellt hat. Positive Erfahrungen liegen dabei vor allem aus dem Bereich des Gewässer- und Wasserschutzes sowie dem Agrarsektor vor. Bereits jetzt ist die Unterstützung durch die Tourismusbank in Österreich ein wichtiges Steuerungsinstrument. Eine gezielte Förderung durch die Österreichische Tourismusbank mit deutlicher Orientierung an den Klimazielen wäre daher eine wichtige Handlungsoption. Die nachstehenden Analysen legen den Schluss nahe, dass sich mit erhöhten Förderungen die notwendigen Veränderungen im eigenen Land schneller erreichen lassen.

Betrachtet man die geplanten Änderungen, die befragte Hotelbetriebe vorgesehen haben (Pröbstl-Haider 2014a), dann beziehen sich diese vermehrt auf die Umrüstung von Heizsystemen, Energiesparmaßnahmen und die Nutzung erneuerbarer Energie (Abb. 13.6 und 13.7). Vor diesem Hin- tergrund wird eine wichtige Handlungsoption darin gesehen, den Tourismusbetrieben in Österreich eine entsprechende Unterstützung für die Umsetzung von Anpassungsmaßnahmen rasch und unkompliziert zukommen zu lassen. Die Erfolge, die in Deutschland mit dem ersten Erneuerbare-EnergienGesetz (EEG) erzielt wurden und dort zu einem enormen Anstieg der Energiegewinnung durch Fotovoltaikanlagen auf privaten Flächen geführt haben, sollten hierzu ebenfalls ermutigen (Wirth 2019).

Unter diesen Voraussetzungen - das heißt einer gezielten und großzügigen staatlichen Förderung privater Initiativen - ließe sich ein inkrementeller Adaptionsprozess erreichen (d. h. eine Anpassung durch viele kleine Schritte), der für den Tourismus bislang als eher wenig vielversprechend eingestuft wurde (Becken 2019). Dies mag auf der globalen touristischen Ebene zutreffen, könnte aber in einem Land wie Österreich, mit starker touristischer Ausrichtung und einer Vielzahl von kleinen bis mittleren Einzelbetrieben, durchaus das richtige Mittel sein und gut umgesetzt werden. Dies 
gilt umso mehr, als in Österreich der Einfluss ausländischen Kapitals im Tourismus noch eine weit geringere Rolle spielt als etwa in der Schweiz, wo seit Langem eine zunehmende Abhängigkeit von Finanzierungen von außerhalb festgestellt und kritisiert wird (Kulmann und Schegg 2012).

\subsubsection{Instrumente der verhaltensbezogenen Ökonomie und Psychologie}

Die Verhaltensökonomik (syn. Verhaltensökonomie oder Wirtschaftspsychologie) ist ein noch relativ junges Forschungsfeld, in der Schnittmenge von Ökonomie und Psychologie. Im Mittelpunkt steht die Auseinandersetzung mit menschlichen Entscheidungen. Untersucht werden psychologische, kognitive, emotionale, kulturelle und soziale Einflussfaktoren wirtschaftlicher Entscheidungen, die einzelne Personen, aber auch Institutionen treffen. Darüber hinaus geht es darum, zu verstehen, warum von erwarteten Entscheidungen oder von Verhalten abgewichen wird, das ,vernünftigerweise“ anzunehmen gewesen wäre (Brekke und Johansson-Stenman 2008; Klege et al. 2018; Stabauer et al. 2018). Die Verhaltensökonomik basiert auf ökonomischem Wissen und integriert dabei psychologische Erkenntnisse. Vielfach geht es in diesem Zusammenhang um die spezifische Abwägung wirtschaftlicher Aspekte mit anderen individuellen Präferenzen, persönlichen Vorurteilen, anderen Eigenschaften eines Produkts oder alternativen Vorgehensweisen. Von der Verhaltensökonomik inspirierte Instrumente zur Beeinflussung von Verhalten (wie hier der Klimawandelanpassung) zielen daher darauf ab, „,das Verhalten der Menschen auf vorhersehbare Weise zu ändern, ohne Optionen zu verbieten oder ihre wirtschaftlichen Anreize wesentlich zu ändern“ (Thaler und Sunstein 2008; Benartzi et al. 2017). Nach Saghai (2013) bieten sich verschiedene verhaltensökonomische Instrumente an, die im Zusammenhang mit der Klimawandelanpassung Anwendung finden könnten. Hierzu gehören die Überprüfung von Handlungsoptionen und Fragestellungen wie: welche Folgen der Verlust von Wahlmöglichkeit hätte, ob und inwieweit die Konfrontation mit ungünstigeren Bedingungen das Verhalten beeinflussen würde oder ob das Auslösen kognitiver Prozesse bei einer Person Verhaltensänderungen bewirken könnte. $\mathrm{Zu}$ den dabei häufig getesteten Auslösern gehören kontrollierende Aktivierungsmechanismen, eher abschreckende (negative) monetäre Anreize, entsprechende positive finanzielle Anreize, aber auch Instrumente, die sich einer rationalen Überzeugungsstrategie bedienen.

$\mathrm{Zu}$ den häufig eingesetzten Forschungsmethoden gehört das Choice-Experiment (diskretes Entscheidungsexperiment oder diskretes Auswahlexperiment), das es ermöglicht, Wahlentscheidungen differenziert zu untersuchen (Lancaster 1971; Louviere et al. 2000). Das Choice-Experiment, das in der Regel in einem Fragebogen zur Anwendung kommt, ist eine Auswahl- bzw. entscheidungsbasierte Methode zur Analyse von
Präferenzen. Die Befragten werden dabei gebeten mehrfach eine Auswahlentscheidung aus verschiedenen Alternativen oder Szenarien zu treffen. Jedes der Szenarien ist durch eine Reihe von Eigenschaften (Attributen) beschrieben. Durch die ökonometrische Analyse dieser vielen Auswahlentscheidungen kann der relative Einfluss jedes Attributs auf das Auswahlverhalten bestimmt werden. Ist eine der Eigenschaften ein zu zahlender Geldbetrag, kann auch die Zahlungsbereitschaft der Befragten abgeschätzt werden. Entwicklungen, wie

- die Notwendigkeit, komplexe Sachverhalte zu untersuchen,

- das Interesse an Entwicklungsoptionen, Szenarien und Risiken sowie

- die Berücksichtigung neuer Unsicherheiten (u. a. im Zusammenhang mit dem Klimawandel),

unterstützen von der inhaltlichen Seite das Interesse an Methoden wie dem Choice-Experiment, das in vielen komplexen Forschungsfeldern zur Anwendung kommt (Bateman et al. 2002; Hensher et al. 2005; de Groot und Hein 2007; PröbstlHaider 2016).

Wenn es darum geht, die Attraktivität neuer Angebote herauszuarbeiten, die Zahlungsbereitschaft von Gästen zu analysieren, den Einfluss von gesellschaftlichen Diskursen oder die Auswirkungen medialer Berichterstattungen zu Risiken und Gefahren beim Urlaub im Berggebiet dazustellen, dann könnten jeweils Methoden der Verhaltensökonomik erfolgreich eingesetzt werden. Die Fragestellungen in Abb. 13.8 und $\mathrm{Abb}$. 13.9 eignen sich besonders gut, um mit diesen Methoden analysiert zu werden.

Mit Beantwortung dieser Fragen würden dann auch die Grundlagen geschaffen, um zu erforschen, wie sich die Treibhausgasbilanz des österreichischen Tourismus durch eine derartige Entwicklung verändern würde.

Ein weiteres Beispiel für den Einsatz von Instrumenten, die Abwägungsentscheidungen beinhalten und die Verhaltenspräferenzen untersuchen, ergibt sich auch im Bereich Wellness, Fitness und Gesundheit. In diesem Bereich ist mit einer zunehmenden Nachfrage zu rechnen, denn bereits heute sind für rund $40 \%$ der Gäste das persönliche Wohlbefinden und entsprechende Angebote zu Bewegung, Entspannung und Wellness ein wichtiger Teil ihrer Reise (Reinhardt 2019).

In diesem Zusammenhang ergeben sich Forschungsfragen im Blick auf die daraus resultierenden Energiekosten, Abwägungsentscheidungen vor allem von kleineren Betrieben und mögliche Anpassungsstrategien nicht nur von Betrieben, sondern auch von Kommunen oder Destinationen. Lösungsansätze könnten in neuen Produktentwicklungen (etwa eine gemeinsame Wellnessanlage für eine Reihe kleinerer Betriebe sowie die einheimische Bevölkerung), der Investition in erneuerbare Energiequellen ebenso bestehen wie in einer Um- 
Abb. 13.8 Methoden der verhaltensbezogenen Ökonomie eignen sich gut im Bereich der Mobilitätsforschung

Was wäre, wenn die Nachfrage nach Anreiseangeboten ohne Auto zunimmt, weil in den Großstädten der Anteil an Personen ohne Auto und ohne Führerschein zunimmt? Derzeit beträgt der Anteil von Haushalten ohne Pkw in Wien bereits 42 \% (VCÖ, 2017) und in Berlin bereits 51 \% (Amt für Statistik Berlin Brandenburg, 2018).

$\rightarrow$ Würden attraktive An- und Abreiseformen für diese Zielgruppe zur Verfügung stehen (z. B. hochrangige Zugverbindungen)?

$\rightarrow$ Wären die Destinationen für eine flexible Mobilität ihrer Gäste gerüstet und hätten sie entsprechende Angebote?

$\rightarrow$ Könnte die Attraktivität von Urlaubsdestinationen durch attraktivere Gestaltung des öffentlichen Raums gesteigert werden?

Auch Fragestellungen zu anderen Formen der Mobilität ließen sich mit dieser Methode gut untersuchen:

Eine wichtige Zielsetzung im Hinblick auf die Einsparung von Treibhausgasemissionen ist es, die Zahl der Reisenden mit Billigflügen zu reduzieren.

$\rightarrow$ Welche Maßnahmen müssen ergriffen werden, um Billigflüge unattraktiv zu machen?

$\rightarrow$ Wie müssen Angebote von Bussen und Bahnen gestaltet werden, dass sie als gleichwertiger Ersatz von den bisherigen Kunden der Billigfluglinien eingestuft werden würden?

$\rightarrow$ Wie unterscheiden sich die Marktsegmente und warum werden bestimmte Angebote von einigen Gästesegmenten stärker angenommen als von anderen?

Abb. 13.9 Unsicherheiten und Risiken müssen mit anderen Eigenschaften der Destination abgewogen werden. Die Verhaltensökonomik ist hierfür ein geeigneter methodischer Ansatz
Was wäre, wenn einzelne Aktivitäten (wie z. B. Klettern im Permafrostbereich, Kanufahren bei Niedrigwasser, Flugsportarten durch veränderte Thermik) gefährlicher werden?

$\rightarrow$ Wie werden diese Risiken vom Gast wahrgenommen?

$\rightarrow$ Wie können diese Risiken als Folge des Klimawandels kommuniziert werden?

$\rightarrow$ Wie beeinflussen Risiken, Unfälle beziehungsweise Gefährdungen und die entsprechende mediale Berichterstattung die Reiseentscheidung und die gewählten Aktivitäten vor Ort?

$\rightarrow$ Sind die Bergrettung und andere österreichische Rettungseinrichtungen auf eine Zunahme der Rettungseinsätze am Berg, in Gewässern und im Zusammenhang mit Flugsportarten, aber auch aufgrund von Hitzestress vorbereitet und welche Art der Rettungs-Infrastruktur wird aus touristischer Sicht erwartet?

$\rightarrow$ Wie und durch welche Strukturen lassen sich vorbeugende Maßnahmen setzen, um einen besseren Schutz zu gewährleisten? legung der Kosten auf den Gast, was jedoch eine Veränderung der Gästesegmente bewirken könnte.

Instrumente der Verhaltensökonomik eignen sich ganz besonders für die Politikberatung und die politische Entscheidungsfindung, da potenzielle Vorgehensweisen und die darauf möglichen Reaktionen vorausgedacht und zielgruppenspezifische Ergebnisse vorgelegt werden können. Methoden, wie zum Beispiel das Choice-Experiment, erhöhen den Einblick in das Zustandekommen und die Einflussfaktoren von Auswahlentscheidungen (Adamowicz et al. 1998). Darüber hinaus konnten van Beukering et al. (2007) und Adamowicz et al. (1998) zeigen, dass auch nichtmarktfähige Eigenschaften, wie das Landschaftsbild, hypothetische Zustände oder Möglichkeiten sehr gut in einer intuitiv verständlichen Form aufbereitet werden können. Auch sogenannte Non-use Values (sog. Nichtgebrauchswerte) lassen sich mit Choice-Experimenten ausgezeichnet untersuchen. Forschungsarbeiten, unter anderem im Zusammenhang mit dem Klimawandel, zeigten weiterhin, dass ein Choice-Experiment auch Variablen ent- halten kann, die Risiken und Unsicherheiten widerspiegeln (Pröbstl-Haider und Haider 2013; Pröbstl-Haider et al. 2016).

Beispiele für die Behandlung von Risiken und Unsicherheiten bestehen unter anderem im Bereich verschiedener sportlicher Outdooraktivitäten (siehe auch Abb. 13.9).

Im Hinblick auf die touristische Forschung ist es wichtig, dass verschiedene Elemente des Produkts, wie die Anreise, die Aktivitäten vor Ort, der Aufenthalt usw., integriert abgefragt werden und nicht - wie bisher meist üblich - getrennt gesehen werden. Eine besonders mühsame Anreise kann beispielsweise den ganzen Aufenthalt überschatten und zukünftige Entscheidungen beeinflussen.

Die Abb. 13.10 zeigt einen Ausschnitt aus einer Studie im Auftrag des Landes Steiermark, bei der die Mobilität vor Ort (Weingartentaxi) im Zusammenhang mit anderen Angeboten der Urlaubsdestination Südsteiermark in einem Choice-Experiment integriert und nicht losgelöst voneinander abgefragt wurden (Pröbstl-Haider et al. 2017a). 


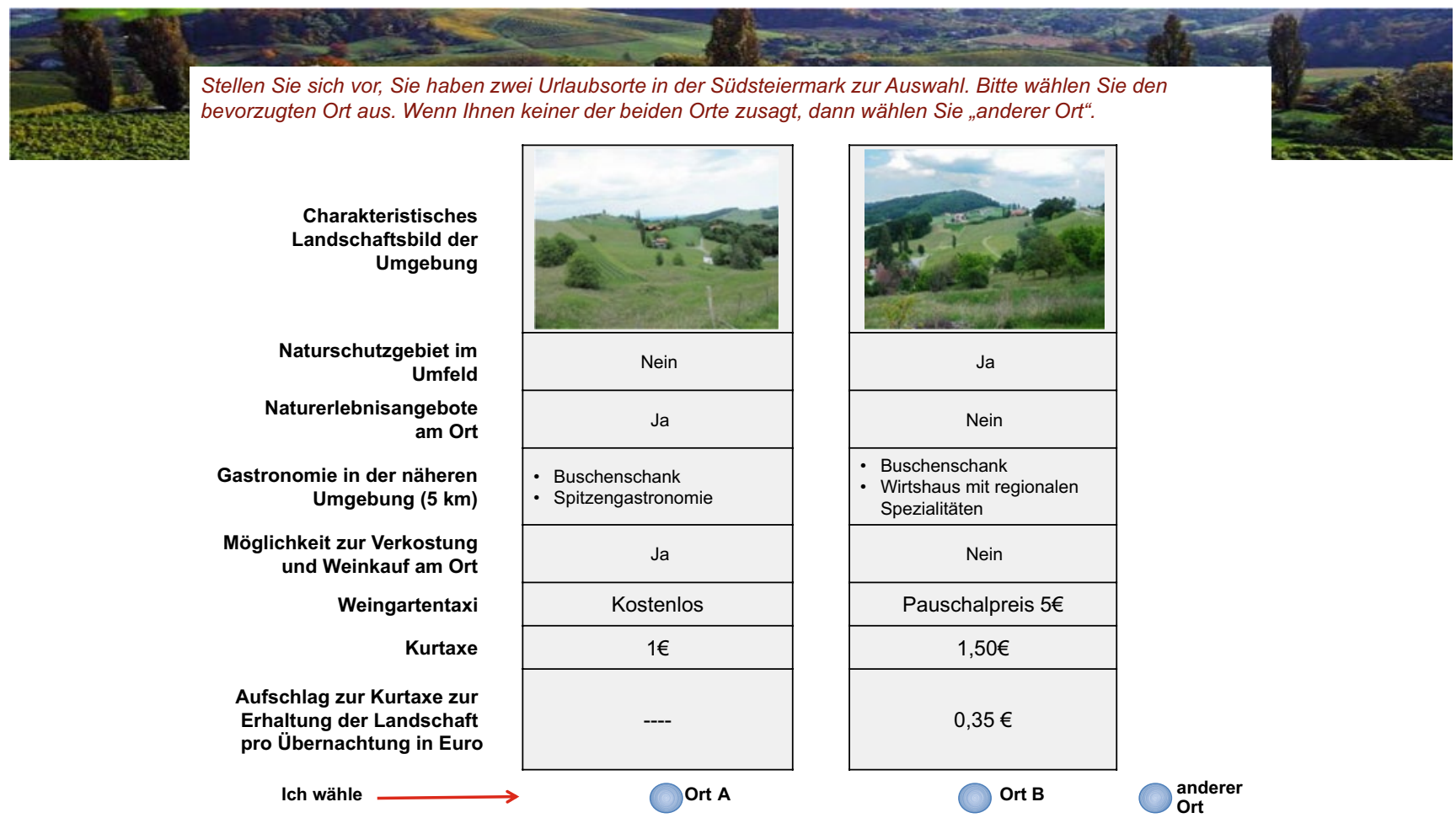

Abb. 13.10 Das Choice-Experiment fragt verschiedene Eigenschaften einer Destination im jeweiligen Kontext ab. Der Befragte muss bis zu sechs solcher Wahlentscheidungen treffen, daraus lassen sich dann praxisnahe Abwägungsentscheidungen und Präferenzen ableiten. (Nachdruck aus Pröbstl-Haider et al. 2017a mit Genehmigung von Springer Nature)

Ein weiteres Argument für einen verstärkten Einsatz verhaltensökonomischer und psychologischer Methoden liegt einerseits im Auseinanderdriften weitreichender technischer Entwicklungen und digitaler Anwendungen und andererseits an der (teilweise mangelnden) Bereitschaft bzw. dem Willen und Wunsch, diese technischen Entwicklungen anzunehmen und im Sinne eines energiesparenden Verhaltens umzusetzen. Becken (2019) fordert in diesem Zusammenhang eine stärkere, integrierte Betrachtung von Technologie und menschlichem Verhalten. So ist zum Beispiel nicht zu erwarten, dass die ältere Generation neue Apps im Bereich der intelligenten Verknüpfung verschiedener Verkehrsmittel nutzen wird bzw. nutzen möchte.

\subsubsection{Fachplanerische Instrumente und Governance}

Die Klimawandelanpassung hat auch einen erheblichen Einfluss auf Planung und Governance, weil die etablierten politischen Strukturen, Prozesse und Instrumente erweitert werden müssen und neue Instrumentarien für innovative Entwicklungen und Anpassungen bestehender politischer Praktiken erforderlich sind. Mit dem Klimawandel steigt der Bedarf an Integration und Koordination zwischen Akteuren, Planungsebenen und fachlichen Sektoren. Im Zusammenhang mit dem Klimawandel ist daher eine Erweiterung der Strukturen der bislang geregelten Planungs- und Beteiligungsformen erforderlich (Knieling und Leal Filho 2013).

Dennoch gelten die spezifischen Fachplanungen, wie Stadt- und Raumplanung, noch immer als entscheidende Instrumente zur Bewältigung der Folgen des Klimawandels, da sie sowohl die Anpassung als auch die Vermeidung betreffen können und wesentlich zur Steuerung der zukünftigen Landnutzung beitragen (Bundesministerium für Nachhaltigkeit und Tourismus 2017). Forschungsprojekte in diesem Zusammenhang zeigten, dass planerische Instrumente weiter greifen können, als nur die Vulnerabilität (Betroffenheit) zum Beispiel aufgrund der naturräumlichen sozialen und wirtschaftlichen Gegebenheiten zu betrachten, sondern über kreative Planungsprozesse weiterreichende Adaptionsprozesse angestoßen werden können (Abb. 13.11). Diese Lösungen können auch dazu führen, dass die geplanten Anpassungsmaßnahmen deutlich über die lokal erkennbare Kapazität zur Anpassung hinausgehen (Pröbstl 2011).

Die große Bedeutung von Planungsprozessen im Zusammenhang mit Tourismus und Klimawandel wird auch darin gesehen, dass insbesondere durch partizipative Planungsprozesse größeren Teilen der Gesellschaft eine Stimme gegeben wird und die Entwicklung dadurch nicht nur durch die touristischen Betriebe oder das Destinationsmanagement bestimmt wird. Dadurch ist die Wahrscheinlichkeit höher, dass ökonomische, ökologische und soziale Belange in gleichberechtigter Weise berücksichtigt werden (Dwyer 2018; Becken 2019). 


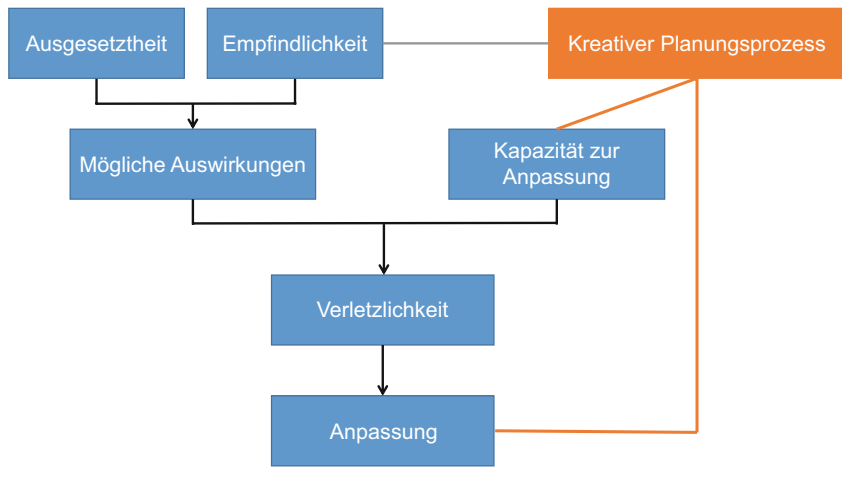

Abb. 13.11 Kreative Planung erlaubt eine Anpassung unabhängig vom Grad der Aussetzung und Verletzlichkeit. (Grafik: Ulrike PröbstlHaider in Pröbstl 2011)

Bei der Anpassung an den Klimawandel ist Innovation, die über Planungs- und Beteiligungsprozesse aktiviert werden kann, von entscheidender Bedeutung. Planungsprozesse haben zudem meist auch die Aufgabe, sektorenübergreifend Anpassungsmaßnahmen und -bedarf gleichzeitig zu berücksichtigen und neben inhaltlichen Aspekten auch die räumliche Perspektive mehr in den Mittelpunkt zu rücken (Strasser et al. 2018; Pröbstl-Haider 2014b). Weiterhin unterstützt Planung die erforderliche sektorenübergreifende räumliche Abwägung, welche die Grundlage für die räumliche Gesamtentwicklung darstellt. Darüber hinaus wird eine proaktive Planung als wichtige Voraussetzung für die Implementierung innovativer und nachhaltiger Technologien, Infrastrukturkonzepte und Marktmodelle angesehen (Strigl et al. 2019; Rehbogen et al. 2020). Planungsprozesse leisten damit einen wesentlichen Beitrag zur Unterstützung, Abstimmung und Kosteneffizienz von Investitionen und Finanzierung von Klimawandelanpassungsmaßnahmen.

Innerhalb der Planungsinstrumente können verpflichtende Instrumente, freiwillig angewandte und von rechtlichen Vorgaben losgelöste Planungsprozesse unterschieden werden. Verpflichtende Planungsinstrumente basieren in der Regel auf rechtlichen Grundlagen. Hierzu zählen unter anderem die Raumordnungsgesetzgebung, die Naturschutzgesetzgebung, aber auch die Gesetzgebung zur Umweltverträglichkeitsprüfung und zur strategischen Umweltprüfung.

Vor allem die Instrumente der Raumplanung, wie der Bebauungsplan, ermöglichen eine proaktive Steuerung. Auch diverse Maßnahmen zur Klimawandelanpassung können darin verpflichtend aufgenommen werden. Dazu gehören Festsetzungen zum Einsatz von erneuerbarer Energie ebenso wie zur Energieversorgung (Busse et al. 2013). Dieses Steuerungsinstrument wird in Österreich, insbesondere im ländlichen Raum, kaum eingesetzt (Stöglehner et al. 2016). Auch Dachund Fassadenbegrünungen und eine angemessene Durchgrünung mit Bäumen und Sträuchern können darüber gesteuert werden. Dies betrifft nicht nur Planungen für die Hotelinfrastruktur, sondern gilt auch für touristische Entwicklungsräume, historische Ortszentren und städtische Destinationen.

Abb. 13.12 zeigt beispielhaft, welche Fragestellungen sich im Zusammenhang mit dem Städtetourismus stellen.

Die Betrachtung der Energieversorgung bereits auf der Bebauungsplanebene könnte nicht nur im Hinblick auf den Bau von und die Umrüstung auf klimaschützende Heizungssysteme relevant sein, sondern aus touristischer Sicht auch im Zusammenhang mit der Klimatisierung von Betrieben.

Diskutiert werden hier Lösungen durch Dämmung, Dachbegrünung und vertikale Begrünung. Eine besondere Herausforderung stellen historische Gebäude dar. ${ }^{7}$

Allen rechtlich verankerten Planungsinstrumentarien gemein sind Abwägungsentscheidungen, zu denen regelmäßig auch der Einfluss des Klimawandels und touristischer Belange, etwa durch das Schutzgut „Mensch-Erholung“ und/ oder die Betrachtung des „Landschaftsbilds“, gehört. Wie wichtig entsprechende raumplanerische Abwägungsprozesse für den Tourismus sind, zeigen Jiricka-Pürrer et al. (2019). Eine Befragung von Sommer- und Wintergästen in Skigebieten zeigte, dass Anpassungsstrategien durch große Windräder und andere großmaßstäbliche Anlagen zur Gewinnung

\footnotetext{
7 Siehe dazu beispielsweise das Interreg-Central-Europe-Projekt BhENEFIT unter https://www.interreg-central.eu/Content.Node/BhENEFIT. html.
}

Abb. 13.12 Planerische Herausforderungen im Zusammenhang mit dem Städtetourismus
Was wäre, wenn die Temperaturen in den Sommermonaten weiter ansteigen und damit den Aufenthalt im Freien sowie charakteristische Produkte (z. B. geführte Stadtrundgänge) einschränken?

$\rightarrow$ Welche Steuerung ist durch raumplanerische Instrumente - insbesondere Durchgrünung und Entsiegelung - möglich?

$\rightarrow$ Welche weiteren Beiträge lassen sich durch Wasserkühlung und andere Infrastruktureinrichtungen erreichen?

$\rightarrow$ Welche Vermeidungsmaßnahmen sind möglich (Verlagerung von Teilen in Gebäude, Bewerbung der kühleren Jahreszeiten)?

$\rightarrow$ Wie kann die Hauptreisezeit für den Städtetourismus in die kühlere Randsaison verlagert werden?

$\rightarrow$ Welche Zielgruppen sind betroffen und welche ökonomischen Einbußen ergeben sich daraus für welche Anbieter? 
erneuerbarer Energie (z. B. Biogasanlage, Fotovoltaik auf Wiesenflächen) aufgrund der Auswirkungen auf das Landschaftsbild und die Atmosphäre des Urlaubsortes in Österreich stark abgelehnt wurden. Anpassungsstrategien in Verbindung mit bestehender Bausubstanz (z. B. Fotovoltaik auf Dächern, Nutzung der Beschneiungsanlage für die Energiegewinnung im Sommer) wurden dagegen besonders begrüßt. $\mathrm{Ob}$ in diesem Zusammenhang die Belange des Tourismus bisher ausreichend berücksichtigt wurden, muss kritisch hinterfragt werden, da Österreich im Gegensatz zu anderen europäischen Mitgliedstaaten Prüfinstrumente und Instrumente zur Vorbereitung von Abwägungsentscheidungen, wie die Umweltverträglichkeitsprüfung oder die strategische Umweltprüfung, sehr begrenzt anwendet (Jiricka und Pröbstl 2008; Stöglehner et al. 2016; Jiricka-Pürrer et al. 2018). Daher besteht die Gefahr, dass touristische Belange nicht oder zu spät Berücksichtigung finden und alternative Lösungen nicht mehr beachtet werden können. Handlungsbedarf wird in einer Verschlankung der Prüfinstrumente bei gleichzeitig breiter Anwendung von harmonisierten Standards gesehen (Pröbstl-Haider 2017).

Für die Anpassung an den Klimawandel spielen darüber hinaus auch planerische Instrumente wie regionale Entwicklungskonzepte, die Raumfunktionen auch mittels Zonierung lenken können, die Entwicklung von Schwerpunkt- und Fokusgebieten für bestimmte Landnutzungen sowie risikobezogene Planungen eine wichtige Rolle (Vetter et al. 2017; Strasser et al. 2018; Rehbogen et al. 2020). Damit diese Instrumente effizient sind, müssen umfassende räumliche Informationen aller relevanten Sektoren verfügbar sein und eine ausreichende Qualität und Aussageschärfe aufweisen. Rehbogen et al. (2020) weisen darauf hin, dass diese Voraussetzungen in einigen, aber nicht in allen Sektoren gegeben sind. Vielfach sind, insbesondere im Bereich Erholung und Tourismus, die räumlichen Grundlagen begrenzt. Landschaftsveränderungen außerhalb der Entwicklung von Infrastruktureinrichtungen und Gebäudeentwicklung werden von behördlicher Seite nicht gezielt erfasst. Damit ergeben sich aus touristischer Sicht Fragestellungen, welche die vielfach hervorgehobene attraktive Struktur der österreichischen Kulturlandschaft und das Landschaftsbild betreffen (PröbstlHaider et al. 2017b).

Entwicklungskonzepte mit Beachtung von Erosions- und Rutschungsrisiken können aber auch im Hinblick auf die Entwicklung der Outdoorinfrastruktur (Wanderwege, Startplätze für Paragliding usw.) unter dem Einfluss des Klimawandels erforderlich werden, um gezielt Adaptions- und Vermeidungsmaßnahmen konzipieren zu können. Dies gilt umso mehr, als die Markierung und Betreuung der Wanderwege in Österreich überwiegend ehrenamtlich erfolgen.

Der Bedarf an freiwilligen, unverbindlichen Planungen und Strategien, die unterschiedliche und sektorenübergreifende Entwicklungsoptionen integriert betrachten, steigt
(Russel et al. 2018; Strasser et al. 2018). Dies zeigt unter anderem der Smart City Masterplan 2025 Salzburg (Stadt Salzburg 2019), der querschnittsorientiert angelegt ist, um eine entsprechend gut fundierte Politik und nachhaltige Abwägung für die verschiedenen Sektoren zu erzielen. Russel et al. (2018) weisen jedoch auch darauf hin, dass die Umsetzung dieser Strategien durch Aktionspläne größtenteils noch aussteht. Ähnliche ganzheitliche Ansätze könnte man sich auch für Destinationen vorstellen.

Engels et al. (2018) betonen auch, dass eine stärkere Verschränkung freiwilliger informeller Pläne mit verpflichtenden formellen Planungen wünschenswert wäre. Dies würde zum Beispiel bedeuten, dass ein informeller Klimamasterplan durch entsprechende Flächenwidmungs- oder Bebauungspläne umgesetzt wird. Insgesamt zeigt sich auch, dass formale Formen der Planung und Beteiligung nicht ausreichen und idealerweise durch informelle ergänzt werden könnten.

\subsubsection{Moderation und Aushandlungsprozesse (Facilitation)}

Der Übergang von planungsbezogenen Governance-Ansätzen in den Bereich der Moderation und Facilitation, die ebenfalls die Umsetzung der Klimapolitik beeinflussen und steuern können, ist fließend (Grotenbreg und van Buuren 2017). Verschiedene Autoren betonen in diesem Zusammenhang die Herausforderung, sektorenübergreifend Akteure einzubinden und polyzentrische Lösungen für eine Klimapolitik im Mehrebenensystem zu entwickeln (Böcher und Nordbeck 2014). Die intensive Beteiligung auf regionaler und lokaler Ebene wird für erforderlich gehalten, um eine mögliche Diskrepanz zwischen Klimaschutzplanungen und deren Umsetzung auf lokaler oder regionaler Ebene möglichst gering zu halten (Carney und Shackley 2009). Die Entwicklung von umsetzungsfähigen Lösungen, bei denen über bestehende Sektoren und Kompetenzfelder hinausgedacht, Funktionen neu definiert und bestehende horizontale und vertikale Abhängigkeiten überwunden werden müssen, erfordern regelmäßig einen erhöhten Koordinierungsaufwand (Bulkeley und Betsill 2013). Deshalb wird im Zusammenhang mit Klimawandelanpassungsprozessen auch die zunehmende Bedeutung von informellen Prozessen und Moderation beschrieben, die einem verstärkten Engagement von lokalen Stakeholdern und Netzwerken Rechnung trägt (Engels et al. 2018). Im Bereich des Tourismus wird ein solcher Koordinierungsbedarf vor allem im Verkehrsbereich beschrieben, wenn es zum Beispiel darum geht, sektorenübergreifende Lösungen für das Problem der „letzten Meile“ oder der lokalen Mobilität zu finden. Die Teilnehmerinnen und Teilnehmer der im Rahmen der Berichtfertigung durchgeführten Workshops mit Stakeholdern betonten, dass ge- 
Abb. 13.13 Bei steigenden Kosten für die Beschneiung sind Aushandlungsprozesse von Bedeutung
Was wäre, wenn die Energiepreise deutlich steigen und die Kosten für flächig beschneite Pisten sich entsprechend erhöhen? Aktuelle Forschungsergebnisse zeigen bereits eine hohe Preissensibilität der Skifahrerinnen und Skifahrer (Pröbstl-Haider \& Flaig, 2019; Pröbstl-Haider \& Mostegl, 2019).

$\rightarrow$ Können die steigenden Kosten für die Beschneiung durch Einkünfte aus der Energieerzeugung zum Beispiel mit Hilfe der Beschneiungsanlage in den Sommermonaten ganz oder teilweise durch die Seilbahn aufgefangen werden?

$\rightarrow$ Können die erhöhten Kosten durch Skipass-Preiserhöhungen aufgefangen werden oder erfordern diese eine Beteiligung zum Beispiel von Nächtigungsbetrieben?

$\rightarrow$ Sind Verlagerungen der Gästeströme in eine in den Hochlagen meist schneereichere Spätwinterzeit (April) möglich? rade in diesem Bereich der Bedarf an moderierten Lösungen besonders hoch ist.

Moderation wird im Gegensatz zur formalen Planung mit festgelegten Beteiligungsprozessen als eher ,weiches“ Instrument zur Förderung der Anpassung angesehen. Die Moderation und Facilitation hilft bestehende Barrieren $\mathrm{zu}$ überwinden, Prozesse zu vereinfachen und Lösungen außerhalb bestehender Strukturen herbeizuführen. Auch wenn - oder vielleicht auch weil - die Ergebnisse des Aushandlungsprozesses rechtlich nicht bindend sind, können sie oftmals mehr bewegen, als wenn verbindliche Instrumente vorgeschrieben worden wären. Nach Grotenbreg und van Buuren (2017) setzt Facilitation darauf, das Eigenengagement nichtstaatlicher Akteure zu ,erleichtern“. Das bedeutet, dass Kompetenzen und Steuerungsaufträge dem Moderationsprozess und der Selbstorganisation gesellschaftlicher Akteure übertragen werden. Entsprechende Lösungen sind immer dann besonders erfolgreich, wenn bestimmte Anforderungen beachtet werden (Danielzyk und Sondermann 2018). Dazu gehören ein hohes Maß an Flexibilität und situationsgerechter Anpassung bei der Vorgehensweise sowie eine gezielte Einbeziehung umsetzungsrelevanter Akteure. Von entscheidender Bedeutung ist auch, dass der Prozess nicht durch zu viele Vorgaben und Wünsche der beteiligten Parteien belastet wird. Im Falle von moderierten und ausverhandelten Lösungen ergibt sich eine Verbindlichkeit in der Regel aus der Selbstbindung der Akteure im Prozess. Dieses Instrument ist jedoch nur dann erfolgreich, wenn der Dialog und die Umsetzungsorientierung im Mittelpunkt stehen und im Vorfeld als Ziel vereinbart wurden. Erfolgreiche Prozesse sind weiterhin durch Verfahrensregeln gekennzeichnet, die sich die beteiligten Akteure oder Stakeholder in der Regel selbst gegeben haben.

In Österreich übernehmen in vergleichbarer Weise zum Beispiel die Klima- und Energiemodellregionen (KEM) und Klimawandelanpassungsregionen (KLAR!) entsprechende Aufgaben. So setzen im Jahr 2019 insgesamt 95 österreichische Klima- und Energiemodellregionen in 819 Gemeinden selbstständig lokal ausverhandelte Klimaschutzprojekte um. Allerdings erwies sich ihr Beitrag im Bereich Tourismus bisher als eher gering, wie eine Analyse von Salak (2016) zeigt. Bei den Klimawandelanpassungsregionen unterstützt der
Klima- und Energiefonds seit 2017 insgesamt 44 Regionen dabei, sich frühzeitig auf die Herausforderungen des Klimawandels einzustellen. Ziel ist es, ein regionales Anpassungskonzept zu entwickeln und anschließend umzusetzen. Auch touristische Belange sollen, wie die Informationsveranstaltungen hierzu zeigen, Berücksichtigung finden. Ergebnisse liegen hierzu jedoch noch nicht vor.

Entsprechende Aushandlungsprozesse könnten erforderlich und erfolgreich eingesetzt werden, wenn es beispielsweise um die Umlegung der Energiekosten für die technische Beschneiung geht (vgl. dazu auch Fragestellungen in Abb. 13.13). Diese stellt zwar eine wichtige technische Anpassung für die Skiregion dar, wird bislang jedoch meist nur von den Bergbahnen getragen, obschon ein erheblicher Nutzen für andere touristische Betriebe, den Einzelhandel u. v. a. besteht.

Auch Lösungen im Zusammenhang mit Hitzeperioden und bestimmten Outdooraktivitäten müssen vor Ort entwickelt werden und erfordern die Zusammenarbeit verschiedener lokaler Akteure.

Die Vorgehensweise und Form der jeweiligen Beteiligungs- und Moderationsprozesse müssen der konkreten Aufgabenstellung angepasst werden. Auch die Mitgestaltungsmöglichkeiten der Stakeholder müssen unter anderem im Zusammenhang mit der Anzahl der Beteiligten, dem zur Verfügung stehenden Zeit- und Kostenrahmen, dem Thema und der Art des zu erzielenden Ergebnisses individuell angepasst werden (Bischoff et al. 2005).

\subsubsection{Bewusstseinsbildung und Information}

Der Weg zum „Paris Lifestyle“ erfordert eine Bewusstseinsänderung als Grundlage für Verhaltensänderungen und Anpassungen. Eine Voraussetzung dafür bildet auch der Zugang zu adäquater, aktueller, vollständiger und leicht verständlicher Information zu diesem Thema. Nach Steurer (2013) stellen Bewusstseinsbildung und Information die Basis für eine sanfte Regulierung und Intervention hin zu Anpassungs- und Vermeidungsmaßnahmen dar. Als wesentliche und erfolgreiche Instrumente gelten in diesem Zusammenhang Unterstützungsbekundungen, Benchmarkingberichte, Broschüren, Leitlinien, Websites und Medienkampagnen. Mostegl (2019) 
Abb. 13.14 Angaben auf Buchungsplattformen könnten einen wichtigen Beitrag zur Information der Reisenden leisten
Was wäre, wenn jede Touristin und jeder Tourist bereits in der Urlaubsplanung über den $\mathrm{CO}_{2}^{-}$ Fußabdruck der geplanten Reise Bescheid wüsste (z. B. durch entsprechendes Labeling auf Buchungsplattformen)?

$\rightarrow$ Würden mehr Urlauberinnen und Urlauber in Österreich bleiben, statt eine Fernreise zu unternehmen?

$\rightarrow$ Was müssten Tourismusdestinationen/Hotellerie-Betriebe tun, um im Wettbewerb hinsichtlich der öffentlichen Erreichbarkeit nicht zu verlieren? Welche Angebote müssen dafür geschaffen werden?

$\rightarrow$ Wie können Tourismusdestinationen/Hotellerie-Betriebe klimabewusste Gäste verstärkt ansprechen und informieren? zählt darüber hinaus auch die Kennzeichnung von Produkten, die Zertifizierung und Bewertung sowie Schulungen und Wettbewerbe ebenfalls zu den Maßnahmen zur Bewusstseinsbildung. Im Allgemeinen zielen alle diese Instrumente darauf $\mathrm{ab}$, den möglichen Einfluss unvollständiger Information $\mathrm{zu}$ korrigieren, um es den Akteuren zu ermöglichen, begründete Entscheidungen zu treffen (Vetter et al. 2017).

Der $\mathrm{CO}_{2}$-Fußabdruck gehört zu den Bereichen, in denen Verbraucher besonders unsicher sind, über dessen Darstellung jedoch viele verhaltensrelevante Informationen vermittelt werden könnten. Ansatzpunkte für eine weiterführende Diskussion hierfür siehe Abb. 13.14.

Bewusstseinsbildung zielt darauf ab, die Ansichten der Bürger zu einem Thema (z. B. Klimawandel) zu formen, ggf. $\mathrm{zu}$ ändern und nachhaltiges Verhalten $\mathrm{zu}$ fördern (Klege et al. 2018). Durch eine erfolgreiche Informationspolitik könnte potenziell auch das Ausmaß zusätzlicher politischer und wirtschaftlicher Maßnahmen sowie sozialer Anpassungsmaßnahmen verringert werden. Darüber hinaus hat sich herausgestellt, dass eine Informationspolitik sinnvoll mit anderen Instrumenten kombiniert werden kann und teilweise auch eine wesentliche Voraussetzung für andere (insbesondere normative und wirtschaftliche) Instrumente darstellt. Vielfach sind normative oder ökonomische Instrumente nur dann verständlich und nachvollziehbar, wenn die Adressaten im Voraus ausreichend informiert werden (Rehbogen et al. 2020).

Zahlreiche Autoren weisen jedoch im Zusammenhang mit dem Klimawandel darauf hin, dass die Kommunikation und Information deswegen erschwert sind, weil Maßnahmen generationsübergreifend wirken und damit die Sensibilisierung eine besondere Herausforderung darstellt. Diverse Studien zeigten, dass vor allem eine zielgruppenspezifische Ansprache erforderlich ist (vgl. Prutsch et al. 2014; Pröbstl-Haider et al. 2018). Insgesamt zählen folgende Aspekte $\mathrm{zu}$ den Erfolgsfaktoren (vgl. Vetter et al. 2017; Klege et al. 2018):

- Kenntnis der ausgewählten Zielgruppe sowie deren aktuelles Verhalten und Vorlieben (im Tourismus insbesondere im Hinblick auf Urlaubersegmente und deren Motive),
- angemessen entwickelte und leicht zugängliche Informationen,

- etablierte und benutzerfreundliche Informationskanäle,

- ansprechende Aufbereitung und Gestaltung zur Förderung der Aufgeschlossenheit der Zielgruppen und Beteiligten gegenüber Verhaltensänderungen,

- ausreichende organisatorische Ressourcen und Glaubwürdigkeit des Informationsanbieters.

Es zeigt sich, dass überregionale Vernetzungen sowie eine europaweite Betrachtung an Bedeutung gewinnen. Beispiel dafür sind die aktuellen Ansätze zur Sensibilisierung und Information auf europäischer Ebene unter anderem im Zusammenhang mit der Unterstützung und Mobilisierung übernationaler Klimanetzwerke, wie den EU-Bürgermeisterpakt für Klima und Energie und den UN-Pakt der Bürgermeister. Im Bereich touristischer Anbieter fehlt diese starke überregionale Initiative. Vielfach ist die Denkweise noch immer eher durch Abgrenzung der Destinationen (national und europaweit) bestimmt. Die Anpassung auf lokaler Ebene wird daher nur langsam in die Beratungsleistungen lokaler Berater und Beratungsverbände einbezogen (Vetter et al. 2017).

Weitere Initiativen werden auch durch eine europäische Richtlinie gefördert (EU-Verordnung 2017/1926). Dies gilt insbesondere im Hinblick auf die Zugänglichkeit zu digitalen Informationen und die Vernetzung von Verkehrsmitteln.

Neben dem Schließen von Informationslücken beziehungsweise dem verbesserten Zugang zu Informationen muss auch ein schrittweiser Bewusstseinswandel bei Anpassungsstrategien im Tourismus mitgedacht werden. Umfragen (z. B. BMUB und UBA 2016) zeigen, dass das klima- und umweltspezifische Problembewusstsein in westlichen Gesellschaften relativ gut ausgeprägt ist. Dies resultiert allerdings nicht immer in ökologischem Handeln (sog. Attitude-BehaviourGap). In diesem Zusammenhang stellen sich für die Branche wichtige Fragen:

- Welche Angebote werden jetzt schon erfolgreich genutzt? Inwieweit müssen bestehende Angebote angepasst 
Abb. 13.15 Ein verändertes Ernährungsbewusstsein beeinflusst die touristischen Produkte

Was wäre, wenn der Anteil der Personen, die fleischlose Kost bevorzugen, bis 2030 auf $33 \%$ ansteigt
(der Anteil liegt aktuell bei ca. 10\%)?
$\rightarrow$ Beeinflusst dieser Trend die Produktion in der heimischen Landwirtschaft und kann der
veränderte Bedarf lokal gedeckt werden?
$\rightarrow$ Hat dies Auswirkungen auf die Logistik (inkl. Verpackung, Lagerung, Transportmittel etc.) wenn
zum Beispiel weniger Gefriergut und mehr Frischprodukte eingesetzt werden?
$\rightarrow$ Welche neuen Rezepte müssen entwickelt werden, um ein attraktives Speisenangebot anbieten
$\quad$ zu können?
$\rightarrow$ Welche Effekte und Wahrnehmungen erzeugt ein entsprechendes Angebot bei den Gästen?
$\rightarrow$ Wird dadurch auch - über den Inlandstourismus - die Gesundheit der Bevölkerung gefördert?

werden, damit diese verstärkt von Konsumentinnen und Konsumenten angenommen werden?

- Wie können touristische Unternehmen dazu motiviert werden, klimaschonende Angebote anzubieten?

- Wie weit können diese alleine auf Freiwilligkeit basieren (Corporate Social Responsibility), wo müssen sie durch Förderungen und Gütesiegel gefördert, gefordert und unterstützt werden und wo sollte der Staat regulierend durch Verbote und Gebote eingreifen?

Die Diskussion mit den Stakeholdern im Rahmen der Berichterstellung zeigte darüber hinaus, dass die Art und die Inhalte der bereitgestellten Information sich ändern müssen. War vor fünf Jahren die Information zum Klimawandel und seinen potenziellen Folgen wichtig, so steht heute die gezielte Information über Adaptation und Mitigation in den jeweiligen Bereichen im Mittelpunkt. Anstelle eines allgemeinen Hinweises auf eine klimaschonende Anreise muss in Zukunft die destinations- und betriebsspezifische Information stehen. Die Einführung der sogenannten Mobilitätsberatung ist ein wichtiger Schritt. Die Beratung kann zum Beispiel das Team an der Rezeption unterstützen und ganz konkret die verfügbaren Angebote für eine klimaschonende Anreise zusammenzustellen und durch Ausflugsangebote für den Gast ergänzen. Nicht nur im Bereich der Mobilität ist eine zusätzliche, maßgeschneiderte Beratung notwendig, sondern auch in anderen Bereichen. Wenn sich unter dem Eindruck der Klimadiskussion, aber auch Informationen zum Tierwohl und der Gesundheitsvorsorge die Einstellung gegenüber der Art und dem Umfang des Fleischkonsums ändert, dann muss der Tourismus auch in diesem Bereich reagieren, diese Bewusstseinsänderung positiv aufgreifen und idealerweise verstärken. Dies kann zum Beispiel durch Schulungen in vegetarischer und veganer Küche für die Betriebe erfolgen. Abb. 13.15 verdeutlicht, welche Herausforderungen sich in diesem Zusammenhang für die Branche ergeben könnten.

Eine Möglichkeit, Nachhaltigkeitsaspekte zu kommunizieren, besteht darin, dem Gast offen Nachhaltigkeitsinformationen zur Verfügung zu stellen. Lee und Oh (2014) geben einen Überblick über Theorien zur Informationsverarbeitung und kategorisieren anhand dieses Frameworks Informationsschilder in Hotelzimmern zur Wiederverwendung von Handtüchern und Bettwäsche. Sie unterscheiden zwischen:

- Framing Theory: Framing bedeutet die Präsentation von Informationen in verschiedenen Wertigkeiten (positiv oder negativ). In Bezug auf Nachhaltigkeitskommunikation kann dies bedeuten, die positiven Auswirkungen, z. B. der Einsparung von Ressourcen, zu betonen. Eine negative Konnotation würde dagegen die negativen Auswirkungen betonen, die ein Verzicht auf Ressourcensparen nach sich ziehen würde.

- Regulatory Focus Theory: Diese Theorie unterscheidet zwischen der Betonung der Förderung bestimmter Ziele (Förderung von Ressourcenschonung) bzw. der Vermeidung negativer Auswirkungen (Vermeidung von Ressourcenverschwendung).

- Construal Level Theory: erklärt den Einfluss der psychologischen Distanz auf die kognitive Deutung eines Umstands. Während Nachrichten auf hoher psychologischer Ebene dabei das „Warum?“' (abstrakt) betonen, fokussieren sich psychologische niederschwellige Botschaften auf das ,Wie?“ (konkret, handlungsorientiert). Ebenso sind niederschwellige Botschaften eher an der Gegenwart orientiert als Botschaften auf höherer Ebene.

- Relevance: Bezieht sich auf den Einfluss des Bezugspunkts. Demnach kann eine Botschaft individuumorientiert (,Mein Verhalten ist gut für mich selbst“) oder gesellschaftsorientiert sein (,Mein Verhalten unterstützt gesellschaftliche Nachhaltigkeitsbestrebungen").

Diese Theorien wurden für experimentelle Tests im Zusammenhang mit der Gästekommunikation und der Entwicklung von nachhaltigen gastronomischen Angeboten zugrunde gelegt (Antonschmidt und Lund-Durlacher 2018). 


\subsection{Zusammenfassung}

Der Tourismus ist sowohl als Verursacher als auch als Betroffener des Klimawandels zu sehen:

\subsubsection{Klimabelastende Wirkung des Tourismus}

Die mit Abstand größten Belastungen im Blick auf die touristischen Treibhausgasemissionen werden durch den Anund Abreiseverkehr sowie, deutlich nachrangiger, durch die Beherbergung und Gastronomie verursacht. Der Aufwand für die Aktivitäten der Gäste - selbst für den Wintersport mit Beschneiung und Pistenpräparierung - ist demgegenüber unbedeutend.

Der Bericht zeigt auf, dass die Anreise nach Österreich überwiegend klimabelastend erfolgt, da drei von vier Urlauberinnen und Urlaubern hierfür den Pkw und rund $10 \%$ das Flugzeug nutzen. Bus und Bahn (mit sehr niedrigen Emissionen) werden nur von einem kleinen Teil der österreichischen Gäste genutzt, während der Flugverkehr und die damit einhergehenden hohen Emissionen vermehrt zunehmen.

Auch die Beherbergungsbetriebe verursachen Treibhausgasemissionen. Die höchsten Energieeinsparpotenziale sind im Bereich der baulichen Substanzen sowie mittels Energieeffizienzmaßnahmen zu erzielen. In diesem Zusammenhang ist auch hervorzuheben, dass die Nachfrage nach Gesundheits- und Wellnesstourismus steigt und auch die hierfür erforderlichen Anlagen einen hohen Energiebedarf aufweisen.

\subsubsection{Erwartete Klimaveränderungen und ihre Auswirkungen auf den Tourismus}

Die Wirkungen des Klimawandels lassen sich an verschiedenen Kenngrößen zweifelsfrei nachweisen. So ist die Lufttemperatur in Österreich seit Ende des 19. Jahrhunderts um knapp $2{ }^{\circ} \mathrm{C}$ etwa doppelt so stark angestiegen wie im globalen Mittel. Ein weiterer Temperaturanstieg von $+1,3{ }^{\circ} \mathrm{C}$ bis zum Jahr 2050 (im Vergleich zum Mittel 1971-2000) ist unabhängig von den verschiedenen Emissionsszenarien zu erwarten. Die Erwärmung geht einher mit einer Zunahme der Hitzebelastung (längere und intensivere Hitzewellen) und häufigeren extremen Dürreereignissen aufgrund ansteigender Verdunstung. Weiterhin haben die natürliche Schneedeckendauer und Schneehöhe vor allem im Westen und Süden Österreichs seit 1950 stark abgenommen. Diese Entwicklung wird sich weiter fortsetzen. Parallel wirkt sich die globale Erwärmung auch auf die Anzahl und Dauer der Zeitfenster für die technische Beschneiung in allen Höhenlagen aus. Die Anzahl der Stunden mit guter Eignung für die technische Beschneiung wird weiter abnehmen.
Weiterhin wird davon ausgegangen, dass ohne Klimaschutzmaßnahmen mit einer deutlichen Zunahme der Intensität und Häufigkeit von kleinräumigen Extremereignissen (Risiko für lokale Starkregen, Überflutungen, Gewitter und Hagel) zu rechnen ist und Folgewirkungen wie Hangrutschungen und Muren eintreten können. Die Folgen dieser Entwicklung zeigen sich in verschiedenen Bereichen:

- Eine hohe Betroffenheit wird, je nach Lage und Ausdehnung, der Städtetourismus erfahren. Für städtische Destinationen werden Hitzewellen und deren gesundheitliche Auswirkungen die größte Herausforderung in den nächsten Jahrzehnten sein.

- Der Einfluss des Klimawandels auf Beherbergungsbetriebe ist bereits heute spürbar. Wetterextreme verringern die Attraktivität von Destinationen, was sich auch negativ auf die Betriebe auswirkt. Klimawandelinduzierte Kostensteigerungen sind zu erwarten, wobei es gleichzeitig auch viele Möglichkeiten gibt, kostenneutral Energie einzusparen.

- Klimabezogene Risiken für Gastronomiebetriebe bestehen vor allem in einer durch Ernteausfälle möglichen Einschränkung der Nahrungsmittelversorgung. Zusätzlich können höhere Kosten durch Importe sowie durch die zur Erreichung der Klimaziele notwendige Besteuerung von Energie beziehungsweise $\mathrm{CO}_{2}$-intensiven Produkten entstehen.

- Im Bereich des Wintertourismus wird davon ausgegangen, dass die beschriebenen Effekte zu räumlichen Umverteilungen führen und den ohnehin steigenden Wettbewerbsdruck verschärfen werden. Dieser Wettbewerb umfasst auch andere (besonders klimaschädliche) Urlaubsoptionen wie Flugreisen in warme Gebiete und Kreuzfahrten.

- Eine detaillierte Betrachtung der Wertschöpfung zeigt auch, dass mögliche wirtschaftliche Verluste durch Schneemangel im Winter durch den Sommerurlaub mit anderen Aktivitäten derzeit nicht ausgeglichen werden können.

Im Bereich der urlaubsrelevanten Aktivitäten im Sommer zeigt sich, dass sich durch klimawandelinduzierte Phänomene eine hohe Betroffenheit für die Sportarten Klettern und Hochtourengehen, Fischen, Golfen sowie für Wasser- und Flugsportarten ergibt. Diese könnten zu einem Destinations- oder Aktivitätenwechsel führen.

\subsubsection{Handlungsoptionen}

Der Entwicklung und Umsetzung von Handlungsoptionen kommt eine besondere Bedeutung zu, da das Ausmaß der 
beschriebenen Klimarisiken in der zweiten Hälfte des Jahrhunderts noch deutlich durch wirksame und rasche globale Klimaschutzmaßnahmen beeinflussbar ist.

- Die Strategien zur Reduktion der Treibhausgasemissionen im Tourismus können sowohl auf der Ebene der Europäischen Kommission als auch der Bundesregierung zu einem prioritären Thema angesetzt werden.

- Die Tourismusstrategien auf europäischer, nationaler und (teilweise) Länderebene beinhalten zwar Maßnahmen zur Emissionsreduktion, eine Weiterentwicklung und Anpassung dieser Strategien erscheint auf der Grundlage des vorliegenden Berichtes sinnvoll.

Im Transport- und Verkehrsbereich könnte zur Umsetzung der dringend erforderlichen Maßnahmen der Einsatz rechtlicher und normativer Instrumente (z. B. gesetzliche Regelungen und Verordnungen) notwendig sein. $\mathrm{Zu}$ den wichtigen Handlungsoptionen gehören in diesem Zusammenhang unter anderem:

- volle Anlastung der Klimakosten bei allen Verkehrsmitteln,

- Bereitstellung von Infrastrukturen für Fahrzeuge mit alternativen Antrieben,

- Maßnahmen zur Klimawandelanpassung der Verkehrsinfrastruktur sowie des Verkehrsmanagements im Krisenfall zur Erhöhung der Resilienz und Sicherstellung der Erreichbarkeiten.

Die gezielte wirtschaftliche Förderung von Klimawandelanpassungsmaßnahmen und der Einsparung von Treibhausgasemissionen wird zukünftig weiter an Bedeutung gewinnen. Nachdem im Tourismussektor vor allem bei der Beherbergung, der Gastronomie und dem Transport ein hohes Potenzial zur Einsparung von Emissionen besteht, würden ökonomische Steuerungsinstrumente hier idealerweise ansetzen:

- Steuerungsmaßnahmen und finanzielle Anreize zu Energie- und Ressourceneinsparung sowie zur Schaffung klimaneutraler Angebote und Innovationen entlang der gesamten touristischen Wertschöpfungskette sollten mit besonderer Beachtung der touristischen Betriebe weiter und noch stärker gefördert werden.

- Im Hinblick auf den Klimaschutz stehen Förderungen zur Reduktion des Energieverbrauchs in der Beherbergung im Vordergrund, vor allem die sehr effektiven Maßnahmen in Bezug auf bauliche Substanzen (Wärmedämmung etc.). Zu beachten ist auch ein stärkerer Energiebedarf durch höheren Kühlungsbedarf in den Sommermonaten, vor allem im Städtetourismus.
- Im Bereich von wellnessorientierten Betrieben könnte der Fokus auf die Förderung energiesparender technischer Maßnahmen sowie auf die Schaffung von Standards zur Sicherstellung eines möglichst sparsamen Verbrauchs gelegt werden.

- In Gastronomiebetrieben könnte der Einsatz innovativer und energieeffizienter Küchentechnik vermehrt gefördert werden, wenn dadurch Treibhausgasemissionen reduziert werden können.

- Weiterhin wäre es möglich, halböffentliche und öffentliche Mobilitätsangebote für An- und Abreise und vor Ort zu fördern, insbesondere solche, die auch von der lokalen Bevölkerung mitgenutzt werden können.

- Im Zusammenhang mit den Aktivitäten könnten Maßnahmen zur Energiegewinnung im Zusammenhang mit Betriebsgebäuden (z. B. Berghütten, Hangar, Golfanlagen) vermehrt gefördert werden.

- Wenn durch den Klimawandel besonders aufwendige Anpassungsmaßnahmen zum Beispiel für den Bau von Pools bei Gewässerproblemen, Wegeneubau und Schutzmaßnahmen im Gebirge erforderlich sind, könnten für besondere Härtefälle Fördermittel zur Verfügung gestellt werden.

Klimawandelanpassung im Tourismus bedeutet auch eine Anpassung des Verhaltens von Touristinnen und Touristen. Daher zählen auch Instrumente der verhaltensbezogenen Ökonomie und Psychologie zu den wesentlichen Handlungsansätzen.

- In diesem Zusammenhang geht es darum, Angebote zu entwickeln und zu präsentieren, die dazu beitragen, dass Tourismusformen gewählt werden, die eine klimaschonende An- und Abreise und Vor-Ort-Mobilität und -Verhalten fördern.

- Bei der Energieeinsparung im Bereich der Beherbergungsbetriebe spielt auch der Faktor „Mensch“ (Personal, Gäste) bei der Umsetzung von klimarelevanten Maßnahmen eine wichtige Rolle. Entsprechende Anreize sind zu entwickeln und einzusetzen.

- Die Entwicklung klimaneutraler Angebote und Innovationen entlang der gesamten touristischen Wertschöpfungskette ist dann erfolgreich, wenn die Gäste in die Entwicklung miteinbezogen werden.

- Die Gäste sowie deren Konsumverhalten spielen bei der Transformation zu klimaschonenden Gastronomieangeboten eine wesentliche Rolle. Präsentationsmöglichkeiten der Speisen und entsprechende Techniken der Verhaltenssteuerung (z. B. Nudging- und Kommunikationstechniken) spielen eine wichtige Rolle und sollen entsprechend vermehrt eingesetzt werden. 
Weitere Handlungsoptionen erfordern planerische Instrumente und moderierte Prozesse. In diesem Zusammenhang spielt nicht nur die Planung in großem räumlichen Maßstab, sondern auch die Entwicklung neuer touristischer Produkte und die kreative Gestaltung einzelner Bausteine eine wichtige Rolle:

- Für den Städtetourismus sind Anpassungsmaßnahmen in der Städteplanung (z. B. zur Reduktion von Hitzeinseln) für die touristische Anpassung und Angebotsentwicklung erforderlich. Verstärkte Kommunikation und Austausch zwischen diesen Sektoren sind dabei wichtig. Dies gilt insbesondere für die Integration mit der Verkehrsplanung beziehungsweise den lokalen Verkehrsbetrieben.

- Ein wichtiges Planungs- und Steuerungselement stellen Zertifizierungen dar. Sie bilden vielfach einen Anreiz für die Umsetzung von klimaschonenden Maßnahmen.

- Ein Wiederaufleben der klassischen „Sommerfrische“ infolge des Klimawandels wäre für Anbieter, ,alpiner Wellness" vorteilhaft. Um am Markt erfolgreich zu sein, sind allerdings eine Differenzierung des Angebots weg von dem starken Fokus aus das Thema Wasser und hin zu einem ganzheitlicheren Wellnessbegriff - sowie Spezialisierung (z. B. auf eine bestimmte Zielgruppe) und Innovation (z. B. Schaffung kreativer Angebote und Packages) notwendig.

- Handlungsoptionen für Gastronomiebetriebe können auch auf einer neuen, innovativen Planung ihres Speisenangebots basieren, bei dem durch verstärkte Nutzung von regionalen, biologisch und saisonal produzierten Lebensmitteln ein größeres Angebot vegetarischer beziehungsweise veganer Speisen erreicht sowie die Reduzierung und Weiterverwertung von Lebensmittel- und Verpackungsabfällen erzielt wird. Dies trägt zu einer Reduktion der Treibhausgase ebenfalls bei.

Von zentraler Bedeutung sind auch die Bewusstseinsbildung und Klimawandelwahrnehmung. Die Fülle der Anpassungsmaßnahmen sollte daher in eine Vision eingebunden werden. Wichtige Handlungsempfehlungen betreffen folgende Bereiche:

- Um die Ziele des Pariser Klimaabkommens zu erreichen, sollte ein möglichst emissionsarmer Lebensstil (,Paris Lifestyle“), inklusive klimaschonenden Reisens, propagiert und entsprechende Angebote sollten geschaffen werden.

- Die Bewusstseinsbildung bei Anbietern und Nachfragenden für verkehrsreduzierende Tourismusformen sollte eine stärkere Beachtung finden.
- Die Steigerung der Akzeptanz von nichtmotorisierten und öffentlichen Mobilitätsangeboten für An- und Abreise und vor Ort sollte durch Bewusstseinsbildung und Information angestrebt werden.

- Eine transparente klimarelevante Information bei der Buchung, aber auch vor und während der Konsumation (z. B. Kennzeichnung des Treibhausgasfußabdrucks für Gerichte) könnte zur Bewusstseinsbildung und Verhaltensänderung beitragen.

- Im Blick auf zahlreiche Aktivitäten im Sommer sollte ein mediales Frühwarnsystem (bezogen auf Hitze, Gewitter und Starkregen) eingerichtet werden, um Anpassungsmaßnahmen der Betriebe und Gäste zu ermöglichen.

Die Mitarbeiterinnen und Mitarbeiter spielen bei der Umsetzung der Maßnahmen eine wichtige Rolle und müssen entsprechend ausgebildet sein. Deshalb sind entsprechende Schulungen und Weiterbildung unumgänglich.

\subsubsection{Forschungsbedarf}

Es ist derzeit noch ungewiss, ob der Klimawandel die Position des österreichischen Tourismus im internationalen Wettbewerb verbessern oder verschlechtern wird. Tendenziell zählt Österreich im Sommer zu den Gewinnern, im Winter zu den Verlierern. Es besteht in verschiedenen Bereichen jedoch Bedarf nach weiterführender Forschung.

- Die Forschungsförderung ist eine wichtige Aufgabe der öffentlichen Hand und sollte im Hinblick auf das Thema Klimawandel und Tourismus durch kompetitive, öffentliche Ausschreibungen gezielt eingesetzt werden.

- Forschungsbedarf besteht für umfassende Studien zu den Treibhausgasemissionen des österreichischen Tourismus sowie der Reisen der Österreicher ins Ausland.

- Forschungsdefizite bestehen im Hinblick auf den Einsatz von Methoden. Dies gilt besonders für die Verhaltensökonomik, die sich ganz besonders für die Politik-, Destinations- und Unternehmensberatung eignet, weil Vorgehensweisen und mögliche Reaktionen vorausgedacht und zielgruppenspezifische Ergebnisse vorgelegt werden können.

- Im Gegensatz zu den Aktivitäten im Winter bestehen bei den Sommeraktivitäten in vielen Bereichen erhebliche Forschungsdefizite.

- Aus dem Report und den vorliegenden Erkenntnissen lassen sich zahlreiche zu vertiefende Forschungsfragestellungen ableiten. Sie bilden eine Grundlage für weitergehende wissenschaftliche Arbeiten in diesem Gebiet. 


\section{Literatur}

Adamowicz, W., Boxall, P., Williams, M. \& Louviere, J. (1998) Stated preference approaches for measuring passive use values: choice experiments and contingent valuation. American Journal of Agricultural Economics 80(1), 64-75. DOI: https://doi.org/10.2307/3180269

Ajzen, I. (1985) From intentions to actions: a theory of planned behavior. In: Kuhl, J. \& Beckman, J. (Hrsg.) Action control: from cognition to behavior, S. 11-39. Springer, Berlin, Deutschland. DOI: https://doi. org/10.1007/978-3-642-69746-3 2

Ajzen, I. (1991) The theory of planned behavior. Organizational Behavior and Human Decision Processes 50(2), 179-210. DOI: https:// doi.org/10.1016/0749-5978(91)90020-T

Amt für Statistik Berlin Brandenburg (2018) Einkommens- und Verbrauchsstichproben: Basisdaten. Amt für Statistik Berlin-Brandenburg, Potsdam, Deutschland. Online unter: https://www. statistik-berlin-brandenburg.de/BasisZeitreiheGrafik/Bas-evs. asp?Ptyp $=300 \&$ Sageb $=63000 \&$ creg $=$ BBB\&anzwer $=5$ (letzter Zugriff: 27.07.2019).

Antonschmidt, H. \& Lund-Durlacher, D. (2018) Can direct communication at the point of consumption reduce the attitude-behavior gap regarding food waste in hotels? In: J. Pearce (Hrsg.) Conference Proceedings of BEST EN Think Tank XVIII: Innovation and Progress in Sustainable Tourism, S. 19-30. James Cook University, Townsville, Australien. Online unter: http://www.besteducationnetwork. org $/$ ? module=file \&act $=$ procFileDownload\&file $\mathrm{srl}=16042 \&$ sid $=3$ be $9 \mathrm{~d} 5 \mathrm{e} 8 \mathrm{fb} 9472514633 \mathrm{cf} 2760319 \mathrm{fdd} \&$ module_srl=879.pdf (letzter Zugriff: 28.05.2020).

APCC (2014) Österreichischer Sachstandsbericht Klimawandel 2014 (AAR14). Austrian Panel on Climate Change (APCC), Verlag der Österreichischen Akademie der Wissenschaften (ÖAW), Wien, Österreich. Online unter: http://www.austriaca.at/APCC_AAR2014.pdf (letzter Zugriff: 24.03.2020).

Baca-Motes, K., Brown, A., Gneezy, A., Keenan, E.A. \& Nelson, L.D. (2013) Commitment and behavior change: evidence from the field. Journal of Consumer Research 39(5), 1070-1084. DOI: https://doi. org/10.1086/667226

Baldwin, R., Cave, M. \& Lodge, M. (2011) Understanding regulation: theory, strategy and practice. Oxford University Press, Oxford, Vereinigtes Königreich.

Bateman, I.J., Carson, R.T., Day, B., Hanemann, W.M., Hanley, N., Hett, T., Jones-Lee, M. \& Loomes, G., Mourato, S., Özdemiroğlu, E., Pearce, D.W., Sugden, R. \& Swanson, J. (2002) Economic valuation with stated preference techniques: a manual. Edward Elgar Publishing, Cheltenham, Vereinigtes Königreich.

Becken, S. (2013) A review of tourism and climate change as an evolving knowledge domain. Tourism Management Perspectives 6, 53-62. DOI: https://doi.org/10.1016/j.tmp.2012.11.006

Becken, S. (2016) Evidence of a low-carbon tourism paradigm? Journal of Sustainable Tourism 25(6), 832-850. DOI: https://doi.org/10.108 $0 / 09669582.2016 .1251446$

Becken, S. (2019) Decarbonising tourism: mission impossible? Tourism Recreation Research 44(4), 419-433. DOI: https://doi.org/10.1080/ 02508281.2019 .1598042

Becken, S. \& Simmons, D. (2008) Using the concept of yield to assess the sustainability of different tourist types. Ecological Economics 67(3), 420-429. DOI: https://doi.org/10.1016/j.ecolecon.2007.12.025

Benartzi, S., Beshears, J., Milkman, K.L., Sunstein, C.R., Thaler, R.H., Shankar, M., Tucker-Ray, W., Congdon, W.J. \& Galig, S. (2017) Should governments invest more in nudging? Psychological Science 28(8), 1041-1055. DOI: https://doi.org/10.1177/0956797617702501

van Beukering, P., Haider, W., Longland, M., Cesar, H., Sablan, J., Shjegstad, S., Beardmore, B., Liu, Y. \& Garces, G.O. (2007) The economic value of Guam's coral reefs (University of Guam Marine Laboratory Technical Report 116). University of Guam, Mangi- lao, Guam. Online unter: https://data.nodc.noaa.gov/coris/library/ NOAA/CRCP/project/1029/econ value guam coral_reefs.pdf (letzter Zugriff: 31.05.2020).

Biesbroek, G.R., Klostermann, J.E.M., Termeer, C.J.A.M. \& Kabat, P. (2013) On the nature of barriers to climate change adaption. Regional Environmental Change 13(5), 1119-1129. DOI: https://doi. org/10.1007/s10113-013-0421-y

Bischoff, A., Selle, K. \& Sinning, H. (2005) Informieren, Beteiligen, Kooperieren: Kommunikation in Planungsprozessen; eine Übersicht zu Formen, Verfahren, Methoden und Techniken. Dortmunder Vertrieb für Bau- und Planungsliteratur, Dortmund, Deutschland.

BMNT (2017) Österreichische Strategie zur Anpassung an den Klimawandel. Teil 1 -Kontext. Bundesministerium für Nachhaltigkeit und Tourismus (BMNT), Wien, Österreich. Online unter: https://www. bmlrt.gv.at/umwelt/klimaschutz/klimapolitik national/anpassungsstrategie/strategie-kontext.html (letzter Zugriff: 24.03.2020).

BMNT \& BMVIT (2018) \#mission2030. Die österreichische Klimaund Energiestrategie. Bundesministerium für Nachhaltigkeit und Tourismus (BMNT) und Bundesministerium für Verkehr, Innovation und Technologie (BMVIT), Wien, Österreich. Online unter: https:// www.bmlrt.gv.at/service/publikationen/umwelt/mission-2030oesterreichische-klima-und-energiestrategie.html (letzter Zugriff: 13.05.2020)

BMUB \& UBA (2016) Umweltbewusstsein in Deutschland 2016. Ergebnisse einer repräsentativen Bevölkerungsumfrage. Bundesministerium für Umwelt, Naturschutz, Bau und Reaktorsicherheit (BMUB), Berlin und Umweltbundesamt (UBA), Dessau-Roßlau, Deutschland. Online unter: https://www.umweltbundesamt.de/sites/ default/files/medien/376/publikationen/umweltbewusstsein_deutschland_2016_bf.pdf (letzter Zugriff: 13.05.2020).

BMWFW, WKO \& ÖHV (2015) Energiemanagement in der Hotellerie und Gastronomie (Leitfaden, 3. überarbeitete Auflage). Bundesministerium für Wissenschaft, Forschung und Wirtschaft (BMWFW), Wirtschaftskammer Österreich (WKO) und Österreichische Hoteliervereinigung (ÖHV), Wien, Österreich. Online unter: https://www. wko.at/branchen/tourismus-freizeitwirtschaft/hotellerie/Energiemanagement-in-Hotellerie-und-Gastronomie.pdf (letzter Zugriff: 28.05.2020)

Böcher, M. \& Nordbeck, R. (2014) Klima-Governance: die Integration und Koordination von Akteuren, Ebenen und Sektoren als klimapolitische Herausforderung. Einführung in den Schwerpunkt. dms - der moderne staat-Zeitschrift für Public Policy, Recht und Management 7(2), 253-268. DOI: https://doi.org/10.3224/dms.v7i2.17316

Boeing, N. (2019) Flugreisen: Verzicht rettet die Welt nicht. ZEIT WISSEN Nr. 3/2019. Online unter: https://www.zeit.de/zeit-wissen/2019/03/flugreisen-klimaschutz-gewissen-co2-emissionen-treibhausgase/komplettansicht (Letzter Zugriff: 31.05.2020).

Bonadonna, A., Giachino, C. \& Truant, E. (2017) Sustainability and mountain tourism: the millennial's perspective. Sustainability 9(7), 1219. DOI:10.3390/su9071219

Bradbury, A., McGimpsey, I. \& Santori, D. (2013) Revising rationality. Journal of Education Policy 28(2), 247-267. DOI: https://doi.org/1 $0.1080 / 02680939.2012 .719638$

Brekke, K. A. \& Johansson-Stenman, O. (2008) The behavioural economics of climate change. Oxford Review of Economic Policy 24(2), 280-297. DOI: https://doi.org/10.1093/oxrep/grn012

Buckley, P. \& Llerena, D. (2018) Demand response as a common pool resource game: nudges versus prices. Working paper GAEL No. 01/2018. Grenoble Applied Economic Laboratory, Frankreich. Online unter: https://hal.archives-ouvertes.fr/hal-01704457/document (letzter Zugriff: 28.05.2020).

Buckley, R., Gretzel, U., Scott, D., Weaver, D. \& Becken, S. (2015) Tourism megatrends. Tourism Recreation Research 40(1), 59-70. DOI: https://doi.org/10.1080/02508281.2015.1005942 
Bulkeley, H. \& Betsill, M.M. (2013) Revisiting the urban politics of climate change. Environmental Politics 22(1), 136-154. DOI: https:// doi.org/10.1080/09644016.2013.755797

Busse, J., Dirnberger, F., Pröbstl-Haider, U. \& Schmid, W. (2013) Die Umweltprüfung in der Gemeinde: mit Ökokonto, Umweltbericht, Artenschutzrecht, Energieplanung und Refinanzierung. Rehm Verlag, Heidelberg, Deutschland.

Butcher, J. (2003) The moralisation of tourism: sun, sand... and saving the world? Routledge, London, Vereinigtes Königreich.

Carney, S. \& Shackley, S. (2009) The greenhouse gas regional inventory project (GRIP): designing and employing a regional greenhouse gas measurement tool for stakeholder use. Energy Policy 37(11), 42934302. DOI: https://doi.org/10.1016/j.enpol.2009.05.028

Danielzyk, R. \& Sondermann, M. (2018) Informelle Planung. In: ARL (Hrsg.) Handwörterbuch der Raumordnung, S. 963-974. Akademie für Raumforschung und Landesplanung (ARL), Hannover, Deutschland. Online unter: https://shop.arl-net.de/media/direct/pdf/ HWB\%202018/Informelle\%20Planung.pdf (28.05.2020).

DIE ZEIT (2019) Reisen ohne Reue? DIE Zeit Nr. 27/2019, Titelthema der Ausgabe vom 27.06.2020.

Dolnicar, S., Knezevic Cvelbar, L. \& Grün, B. (2017a) Do pro-environmental appeals trigger pro-environmental behavior in hotel guests? Journal of Travel Research 56(8), 988-997. DOI: https:// doi.org/10.1177/0047287516678089

Dolnicar, S., Knezevic Cvelbar, L. \& Grün, B. (2017b) A sharing-based approach to enticing tourists to behave more environmentally friendly. Journal of Travel Research 58(2), 241-252.DOI: https:// doi.org/10.1177/0047287517746013

Dolnicar, S., Knezevic Cvelbar, L. \& Grün, B. (2018) Changing service settings for the environment: how to reduce negative environmental impacts without sacrificing tourist satisfaction. Annals of Tourism Research 76, 301-304. DOI: https://doi.org/10.1016/j. annals.2018.08.003

Dwyer, L. (2018) Saluting while the ship sinks: the necessity for tourism paradigm change. Journal of Sustainable Tourism 26(1), 29-48. DOI: https://doi.org/10.1080/09669582.2017.1308372

EEA (2014) National adaption policy processes in European countries - 2014 (EEA Report No 4/2014). European Environment Agency (EEA), Kopenhagen, Dänemark. Online unter: https://www.eea. europa.eu/publications/national-adaptation-policy-processes (letzter Zugriff: 29.05.2020).

EIW (2014) Energieinstitut der Wirtschaft: eine Zwischenbilanz. EIWInsights 1/2014. Energieinstitut der Wirtschaft GmbH, Wien, Österreich. Online unter: https:/www.energieinstitut.net/sites/default/ files/eiw insights_14_01_eiw_kl_0.pdf(letzter Zugriff: 28.07.2019).

Engels, A., Wickel, M., Knieling, J., Kretschmann, N. \& Walz, K. (2018) Lokale Klima-Governance im Mehrebenensystem: formale und informelle Regelungsformen. In: von Storch, H., Meinke, I. \& Claußen, M. (Hrsg.) Hamburger Klimabericht: Wissen über Klima, Klimawandel und Auswirkungen in Hamburg und Norddeutschland, 265-282. Springer Spektrum, Berlin, Deutschland. DOI: https://doi. org/10.1007/978-3-662-55379-4

EU (2017) Delegierte Verordnung (EU) 2017/1926 der Kommission vom 31. Mai 2017 zur Ergänzung der Richtlinie 2010/40/EU des Europäischen Parlaments und des Rates hinsichtlich der Bereitstellung EU-weiter multimodaler Reiseinformationsdienste. Amtsblatt der Europäischen Union Nr. L 272 vom 21.10.2017. Online unter: https://eur-lex.europa.eu/legal-content/DE/TXT/PDF/?uri=CELEX:32017R1926\&from=DE (letzter Zugriff: 29.05.2020).

Fürnweger, E. (2016) EMAS-Zertifizierung von Golfplätzen. Masterarbeit am Institut für Landschaftsentwicklung, Erholungs- und Naturschutzplanung (ILEN), Universität für Bodenkultur Wien. Online unter: https://abstracts.boku.ac.at/download.php?dataset_id=14295\&property_id=107 (letzter Zugriff: 25.05.2020).

de Groot, R.S. \& Hein, L. (2007) Concept and valuation of landscape functions at different scales. In: Mander, Ü., Wiggering, H. \& Hel- ming, K. (Hrsg.) Multifunctional land use: meeting future demands for landscape goods and services, S. 15-36. Springer, Berlin, Deutschland. DOI: https://doi.org/10.1007/978-3-540-36763-5_2

Gössling, S. \& Peeters, P. (2015) Assessing tourism's global environment impact 1900-2050. Journal of Sustainable Tourism (23)5, 1-21. DOI: https://doi.org/10.1080/09669582.2015.1008500

Grotenbreg, S. \& van Buuren, A. (2017) Facilitation as a governance strategy: unravelling governments' facilitation frames. Sustainability 9(1), 160. DOI: https://doi.org/10.3390/su9010160

Grothmann, T. \& Patt, A. (2005) Adaptive capacity and human cognition: the process of individual adaptation to climate change. Global Environmental Change 15(3), 199-213. DOI: https://doi. org/10.1016/j.gloenvcha.2005.01.002

Grothmann, T., Nenz, D. \& Pütz, M. (2009) Adaptation in vulnerable alpine regions - lessons learnt from regional case studies. In: EEA (Hrsg.) Regional climate change and adaptation: the Alps facing the challenge of changing water resources (EEA Report No 8/2009), S. 96-108. European Environment Agency (EEA), Kopenhagen, Dänemark. Online unter: https://www.eea.europa.eu/publications/alpsclimate-change-and-adaptation-2009 (letzter Zugriff: 29.05.2020).

Hardeman, W., Johnston, M.M., Johnston, D.M, Bonetti, D., Wareham, N. \& Kinmonth, A.L. (2002) Application of the theory of planned behavior in behavior change interventions: a systematic review. Psychology \& Health 17(2), 123-158. DOI: https://doi.org/ 10.1080/08870440290013644a

Hensher, D.A., Rose, J.M. \& Greene, W.H. (2005) Applied choice analysis: a primer. Cambridge University Press, Cambridge, Vereinigtes Königreich.

Holloway, J.C. \& Robinson, C. (1995) Marketing for tourism. Longman, Harlow, Vereinigtes Königreich.

Hunter, C. \& Shaw, J. (2006) Applying the ecological footprint to ecotourism scenarios. Environmental Conservation 32(4), 294-304. DOI: https://doi.org/10.1017/S0376892906002591

Jiricka, A. \& Pröbstl, U. (2008) SEA in local land use planning: first experience in the Alpine States. Environmental Impact Assessment Review 28(4-5), 328-337. DOI: https://doi.org/10.1016/j. eiar.2007.05.002

Jiricka-Pürrer, A., Bösch, M., Pröbstl-Haider, U. (2018) Desired but neglected: investigating the consideration of alternatives in Austrian EIA and SEA practice. Sustainability 10(10), 3680. DOI: https://doi. org/10.3390/su10103680

Jiricka-Pürrer, A., Schmied, J. \& Pröbstl-Haider, U. (2019) Preferences for renewable energy sources among tourists in the European Alps. In: Pröbstl-Haider, U., Richins, H. \& Türk, S. (Hrsg.) Winter tourism: trends and challenges, S. 400-424. CABI, Wallingford, Vereingtes Königreich.

Juvan, E., Grün, B. \& Dolnicar, S. (2018) Biting off more than they can chew: food waste at hotel breakfast buffets. Journal of Travel Research 57(2), 232-242. DOI: https://doi.org/10.1177/0047287516688321

Klege, R., Visser, M., Datta, S. \& Darling, M (2018) The power of nudging: using feedback, competition and responsibility assignment to save electricity in a non-residental setting. ERSA Working Paper, No. 763. Online unter: https://econrsa.org/system/files/publications/working_papers/working_paper_763.pdf (letzter Zugriff: 04.11.2019).

klimaaktiv (2016) Mit klimaaktiv Silber zur TOP-Tourismus-Förderung für Hotelgebäude. Bundesministerium für Klimaschutz, Umwelt, Energie, Mobilität, Innovation und Technologie (BMK), Wien, Österreich. Online unter: https://www.klimaaktiv.at/bauen-sanieren/ dienstleistungsgeb/tourismus-foerderung.html (letzter Zugriff: 29.05.2020).

Knieling, J. \& Leal Filho, W. (2013) Climate change governance: the challenge for politics and public administration, enterprises and civil society. In: Knieling, J. \& Leal Filho, W. (Hrsg.) Climate change governance, S. 1-5. Springer, Berlin, Deutschland. DOI: https://doi. org/10.1007/978-3-642-29831-8_1 
Komppula, R. (2001) New product development in tourism companies: case studies on nature based activity operators. $10^{\text {th }}$ Nordic Tourism Research Symposium, 18.-20. Oktober 2001, Vasa, Finnland. Online unter: http://citeseerx.ist.psu.edu/viewdoc/download?doi=10.1.1.461.8918\&rep=rep1\&type=pdf (letzter Zugriff: 29.07.2019).

Kosters, M. \& van der Heijden, J. (2015) From mechanism to virtue: evaluating nudge-theory. Evaluation 21(3), 276-291. DOI: https:// doi.org/10.1177/1356389015590218

Kullmann, P. \& Schegg, R. (2012) Schweizer Hotellerie. ITO - Institut für Tourismus, HES-SO Valais-Wallis, Sitten, Schweiz. Online unter: https://www.tourobs.ch/media/46746/schweizer-hotellerie-_-publikationen-2012-_-kullmannschegg.pdf (letzter Zugriff: 29.05.2020).

Lancaster, K. (1971) Consumer demand: a new approach. Columbia Univeristy Press, New York, NY, USA.

Lee, S. \& Oh, H. (2014) Effective communication strategies for hotel guests' green behavior. Cornell Hospitality Quarterly 55(1), 52-63. DOI: https://doi.org/10.1177/1938965513504029

Levi-Faur, D. (2011) Handbook on the politics of regulation. Edward Elgar Publishing, Cheltenham, Vereinigtes Königreich.

Loer, K. (2015) „Nudging individual health?“ Neue Perspektiven auf die Gesundheitspolitik. SSOAR Working Paper, GESIS - Leibniz-Institut für Sozialwissenschaften. Online unter: https://nbn-resolving. org/urn:nbn:de:0168-ssoar-51836-8 (letzter Zugriff: 29.05.2020).

Louviere, J.J., Hensher, D.A. \& Swait, J.D. (2000) Stated choice methods: analysis and applications. Cambridge University Press, Cambridge, Vereinigtes Königreich.

Mostegl, N. (2020) Understanding and steering climate change adaptation behaviour. Dissertation, Universität für Bodenkultur Wien.

Mundt, J.W. (2011) Tourism and sustainable development: reconsidering a concept of vague policies. Erich Schmidt Verlag, Berlin, Deutschland.

Njoroge, J.M. (2015) Climate change and tourism adaptation: literature review. Tourism and Hospitality Management 21(1), 95-108. Online unter: https://thm.fthm.hr/past-issues/send/11-vol21no1/45-climatechange-and-tourism-adaptation-literature-review (letzter Zugriff: 29.05.2020).

NPE (2018) Fortschrittsbericht 2018 - Markthochlaufphase: Nationale Plattform Elektromobilität (NPE). Gemeinsame Geschäftsstelle Elektromobilität der Bundesregierung (GGEMO), Berlin, Deutschland. Online unter: http://nationale-plattform-elektromobilitaet.de/ fileadmin/user_upload/Redaktion/NPE_Fortschrittsbericht_2018 barrierefrei.pdf (letzter Zugriff: 28.07. 2019).

Poon, A. (2003) Competitive strategies for a "new tourism”. In: Cooper, C. (Hrsg.) Classic reviews in tourism, S. 130-142. Channel View Publications, Clevedon, Vereinigtes Königreich.

Pröbstl, U. (2011) Herausforderungen des Klimawandels für den Tourismus in der Region Liezen. Handlungsmöglichkeiten von Tourismus und Raumplanung. CLISP - Climate Change Adaptation by Spatial Planning in the Alpine Area (Model Region Report Styria). OIR, ÖIR-Projekthaus, TerraConsult und Rosinak \& Partner, Wien, Österreich.

Pröbstl, U. \& Müller, F. (2012) Hotel certification and its relevance for sustainable development: examples from the European Alps. In: Pineda, F.D. \& Brebbia, C.A. (Hrsg.) Sustainable Tourism V, S. 3-15. WIT Press, Ashurst, Vereinigtes Königreich. DOI: https:// doi.org/10.2495/ST120011

Pröbstl-Haider, U. (2014a) Strategien für den Einsatz erneuerbarer Energien in Hotelbetrieben. Befragung in Zusammenarbeit mit der Österreichischen Hoteliervereinigung (ÖHV). Präsentation ausgewählter Ergebnisse online unter: https://docplayer.org/16454701Einsatz-erneuerbarer-energien-in-hotelbetrieben.html (letzter Zugriff: 29.05.2020).

Pröbstl-Haider, U. (2014b) Berücksichtigung des Klimawandels bei kommunaler Planung und im Umweltbericht. UVP-report 28(1), $14-17$.
Pröbstl-Haider, U. (2016) Aufgaben und Bedeutung des Choice Experiments für die naturtouristische Forschung. In: Mayer, M. \& Job, H. (Hrsg.) Naturtourismus - Chancen und Herausforderungen: Studien zur Freizeit- und TourismusforschungBand 12, S. 17-34. Verlag MetaGIS-Systems, Mannheim, Deutschland.

Pröbstl-Haider, U. (2017) UVP-Verfahren im Vergleich: Unterschiede im Vollzug Österreich und Bayern am Beispiel von Skianlagen. In: Furherr, E. (Hrsg.) Verwaltungsreform im Anlagenrecht: Praxisanalyse der Novellen zur GewO und zum UVP-G, S. 39-50. Facultas, Wien.

Pröbstl-Haider, U. \& Flaig, R. (2019) The knockout deal - pricing strategies in Alpine ski resorts. In: Pröbstl-Haider, U., Richins, H. \& Türk, S. (Hrsg.) Winter tourism: trends and challenges, S. 116-137. CABI, Wallingford, Vereinigtes Königreich.

Pröbstl-Haider, U. \& Haider, W. (2013) Tools for measuring the intention for adapting to climate change by winter tourists: some thoughts on consumer behavior research and an empirical example. Tourism Review 68(2), 44-55. DOI: https://doi.org/10.1108/TR-04-2013-0015

Pröbstl-Haider, U. \& Mostegl, N. (2016) Merging of ski areas: the key concept to attract more winter tourists? In: AIEST (Hrsg.) Tourism at and on the sea, AIEST's advances in tourism research-perspectives of actors, institutions and systems. Abstract Book. International Association of Scientific Experts in Tourism (AIEST), 66th AIEST Conference, Malta.

Pröbstl-Haider, U. \& Mostegl, N. (2019) A matter of culture: how cultural differences shape skiing motivation, behaviour and destination choice. In: Pröbstl-Haider, U., Richins, H. \& Türk, S. (Hrsg.) Winter tourism: trends and challenges, S. 192-211. CABI, Wallingford, Vereinigtes Königreich.

Pröbstl-Haider, U., Mostegl, N., Kelemen-Finan, J., Haider, W., Formayer, H., Kantelhardt, J., Moser, T., Kapfer, M. \& Trenholm, R. (2016) Farmer's preferences for future agricultural land use under the consideration of climate change. Environmental Management 58(3), 446-464. DOI: https://doi.org/10.1007/s00267-016-0720-4

Pröbstl-Haider, U., Haider, W. \& Mostegl, N. (2017a) Tourismus und Weinbau im Naturpark Südsteiermark in Österreich/Nature Park „Südsteiermark“, Austria: Tourism and Viniculture. In: Wagner, D., Mair, M., Stöckl, A. \& Dreyer, A. (Hrsg.) Kulinarischer Tourismus und Weintourismus, S. 145-156. Springer Gabler, Wiesbaden, Deutschland. DOI: https://doi.org/10.1007/978-3-658-13732-8_12

Pröbstl-Haider, U., Mostegl, N.M., Jandl, R., Formayer, H., Haider, W., Pukall, K. \& Melzer, V. (2017b) Bereitschaft zur Klimawandelanpassung durch Kleinwaldbesitzer in Österreich. Allgemeine Forstund Jagdzeitung 188(7-8), 113-126. DOI: https://doi.org/10.23765/ afjz0002007

Pröbstl-Haider, U., Brom, M., Dorsch, C. \& Jiricka-Pürrer, A. (2018) Umweltmanagement in Skigebieten. Springer Spektrum, Berlin, Deutschland. DOI: https://doi.org/10.1007/978-3-662-55988-8

Prutsch, A., Glas, N., Grothmann, T., Wirth, V., Dreiseitl-Wanschura, B., Gartlacher, S., Lorenz, F. \& Gerlich, W. (2014) Klimawandel findet statt...Anpassung ist nötig! Ein Leitfaden zur erfolgreichen Kommunikation. Umweltbundesamt $\mathrm{GmbH}$, Wien, Österreich. Online unter: https://www.umweltbundesamt.at/fileadmin/site/publikationen/DP144.pdf (letzter zugriff: 29.05.2020).

Pütz, M., Gallati, D., Kytzia, S., Elsasser, H., Lardelli, C., Teich, M., Waltert, F. \& Rixen, C. (2011) Winter tourism, climate change, and snowmaking in the Swiss Alps: tourists' attitudes and regional economic impacts. Mountain Research and Development 31(4), 357362. DOI: https://doi.org/10.1659/MRD-JOURNAL-D-11-00039.1

Rapp, C. (2017) Studie zur Wiederverwendung von Hotel-Handtüchern: Macht der Gewohnheit spart Wäsche und schont Umwelt. TUI AG, Hannover, Deutschland. Online unter: https://www.tuigroup.com/ de-de/medien/presseinformationen/ag-meldungen/2017/2017-0808-studie-zur-wiederverwendung-von-hotel-handtuechern (letzter Zugriff: 27.07.2019). 
Rehbogen, A., Edtmayer, H., Leusbrock, I., \& Mauthner, F. (2020) Räumliche Energieplanung für die Wärmewende. TGA Planung 2020, 70-73. Online unter: https://imgs.tga.at/e/80_657f31e3409c e8dd848990a7f6231c4a3a5f7a74.pdf (letzter Zugriff: 29.05.2020).

Reinhardt, U. (2019) Tourismusanalyse 2019. Stiftung für Zukunftsfragen, Hamburg, Deutschland. Online unter: https://www.stiftungfuerzukunftsfragen.de/fileadmin/user_upload/tourismusanalyse/2019/ Stiftung-fuer-Zukunftsfragen-Tourismusanalyse-2019.pdf (letzter Zugriff: 01.06.2020).

Reschl, J. (2019) Umweltkennzeichnungsprogramme im Kontext nachhaltigen Konsums: eine Analyse am Beispiel des Österreichischen Umweltzeichens für Tourismusbetriebe. Masterarbeit am Institut für Landschaftsentwicklung, Erholungs- und Naturschutzplanung (ILEN), Universität für Bodenkultur Wien, Österreich.

Russel, D., Beck, S., Campos, I., Capriolo, A., Castellari, S., den Uyl, R.M., Gebhardt, O., Hildén, M., Jensen, A., Karali, E., Mäkinen, K., McGlade, K., Nielsen, H.Ø., Penha-Lopes, G., Rendón, O., Tröltzsch, J. \& Weiland, S. (2018) Analyzing the policy framework for climate change adaption. In: Sanderson, H., Hilden, M., Russel, D., Penha-Lopes, G. \& Capriolo, A. (Hrsg.) Adapting to climate change in Europe: exploring sustainable pathways - from local measures to wider policies, S. 273-313. Elsevier, Amsterdam, Niederlande. DOI: https://doi.org/10.1016/B978-0-12-849887-3.00006-X

Saghai, Y. (2013) Salvaging the concept of nudge. Journal of Medical Ethics 39(8), 487-493. DOI: https://doi.org/10.1136/medethics-2012-100727

Salak, B. (2016) Energieregionen und Energietourismus: Bedeutung und Chancen im Rahmen der Klima- und Energie-Modellregionen in Österreich. Dissertation, Universität für Bodenkultur Wien, Österreich.

Schenker, O., Mennel, T., Osberghaus, D., Ekinci, B., Hengesbach, C., Sandkamp, A., Kind, C., Savelsberg, J., Kahlenborn, W., Buth, M., Peters, M. \& Steyer, S. (2014) Ökonomie des Klimawandels: integrierte ökonomische Bewertung der Instrumente zur Anpassung an den Klimawandel. Umweltbundesamt, Dessau-Roßlau, Deutschland. Online unter: https://www.umweltbundesamt.de/sites/default/files/ medien/378/publikationen/climate_change_16_2014_oekonomie des klimawandels.pdf (letzter Zugriff: 31.05 .2020 ).

Schmied, J. (2012) Acceptance of infrastructure for renewable energy in alpine destinations by tourists. Masterarbeit am Institut für Landschaftsentwicklung, Erholungs- und Naturschutzplanung (ILEN), Universität für Bodenkultur Wien, Österreich. Abstract online unter: https://abstracts.boku.ac.at/oe_list.php?paID=3\&paLIST=0\&paSID $=10627$ (letzter Zugriff: 31.05.2020).

Schwarzinger, S., Bird, D.N. \& Hadler, M. (2018) The "Paris Lifestyle" - bridging the gap between science and communication by analysing and quantifying the role of target groups for climate change mitigation and adaptation: an interdisciplinary approach. In: Leal Filho, W., Lackner, B. \& McGhie, H. (Hrsg.) Addressing the challenges in communicating climate change across various audiences, S. 375397. Springer, Cham, Schweiz. DOI: https://doi.org/10.1007/9783-319-98294-6_23

Sharpley, R. (2006) Travel and tourism. Sage Publications, London, Vereinigtes Königreich. DOI: https://doi.org/10.4135/9781446213810

Simmons, D. \& Becken, S. (2004) The cost of getting there: impacts of travel to ecotourism destinations. In: Buckley, R. (Hrsg.) Environmental impacts of ecotourism, S. 15-23. CABI, Wallingford, Vereingtes Königreich.

Stabauer, P., Huemer, F., Layer-Wagner, T., Mostegl, N.M., Ngai, E. \& Rubbestad Lilja, J. (2018) SimpliCITY - Marketplace for usercentered sustainability services plattform. Projektantrag, 1. Call "Making Cities Work", Programm "JPI Urban Europe Innovation Action”. Salzburg, Österreich.

Stadt Salzburg (2019) Smart City Salzburg Masterplan 2025: Klimaund Energielösungen für die Zukunft (3. überarbeitete und ergänzte Auflage). Stadt Salzburg Magistrat, MA 6/00 Baudirektion, Salzburg,
Österreich. Online unter: https://www.stadt-salzburg.at/pdf/masterplan_2025_der_stadt_salzburg.pdf (letzter Zugriff: 31.05.2020).

Statistik Austria (2019) Ankünfte und Nächtigungen nach Herkunftsländern, Kalenderjahr 2018. Bundesanstalt Statistik Österreich, Wien, Österreich. Online unter: https://www.statistik.at/web_de/statistiken/ wirtschaft/tourismus/beherbergung/ankuenfte_naechtigungen/index. html (letzter Zugriff: 14.05.2019)

Stehr, N. \& von Storch, H. (1995) The social construct of climate and climate change. Climate Research 5(2), 99-105. DOI: https://doi. org/10.3354/cr005099

Steurer, R. (2013) Disentangling governance: a synoptic view of regulation by government, business and civil society. Policy Sciences 46(4), 387-410. DOI: https://doi.org/10.1007/s11077-013-9177-y

Stöglehner, G., Neugebauer, G., Erker, S. \& Narodoslawsky, M. (2016) Integrated spatial and energy planning: supporting climate protection and the energy turn with means of spatial planning. Springer, Cham, Schweiz. DOI: https://doi.org/10.1007/978-3-319-31870-7

Strasser, H., Kimman, J., Koch, A., Mair am Tinkhof, O., Müller, D., Schiefelbein, J. \& Slotterback, C. (2018) IEA EBC annex 63: implementation of energy strategies in communities. Energy and Buildings 158(1), 123-134. DOI: https://doi.org/10.1016/j.enbuild.2017.08.051

Strigl, A., Rogalli, T., Homeier, I., Pangerl, E., Tollmann, J., Strasser, H., Mostegl, N. \& Rehbogen, A. (2019) transAT: Top-down Instrumente für die Energiewende 2050in Österreich (Langfassung). Endbericht für das Programm Energy Transition 2050. Österreichisches Institut für Nachhaltige Entwicklung (ÖIN), Salzburger Institut für Raumordnung und Wohnen (SIR) und Stadt Wien (MA18: Stadtentwicklung und Stadtplanung), Österreich. Online unter: https://energytransition.klimafonds.gv.at/wp-content/uploads/sites/7/2020/01/ Endbericht_EnergyTransition_final.pdf (letzter Zugriff: 31.05.2020).

Thaler, R. \& Sunstein, C. (2008) Nudge: improving decisions about health, wealth and happiness. Yale University Press, New Haven, CT, USA.

Thorun, C., Diels, J., Vetter, M., Reisch, L., Bernauer, M., Micklitz, H.-W., Purnhagen, K., Rosenow, J. \& Forster, D. (2017) NudgeAnsätze beim nachhaltigen Konsum: Ermittlung und Entwicklung von Maßnahmen zum ,Anstoßen “ nachhaltiger Konsummuster (Abschlussbericht). Umweltbundesamt, Dessau-Roßlau, Deutschland. Online unter: https://www.umweltbundesamt.de/sites/default/files/ medien/1410/publikationen/2017-08-22_texte_69-2017_nudgeansaetze_nach-konsum_0.pdf (letzter Zugriff: 31.05.2020).

TU Graz (o.J.) Fußabdruckrechner. Ökologischer Fußabdruck für Urlaub und Reise. Institut für Prozess- und Partikeltechnik, Technische Universität Graz (TU Graz), Österreich. Online unter: http://www. fussabdrucksrechner.at/de/calculation (letzter Zugriff: 31.05.2020).

VCÖ (2017) VCÖ: Mehr als 850.000 Haushalte in Österreich sind ohne eigenes Auto mobil. VCÖ - Mobilität mit Zukunft, Wien. Online unter: https://www.vcoe.at/news/details/vcoe-mehr-als-850-000haushalte-in-oesterreich-sind-ohne-eigenes-auto-mobil (letzter $\mathrm{Zu}$ griff: 27.07.2019).

VCÖ (2019) Adieu Erdöl! Die Energiezukunft ist erneuerbar. VCÖ Magazin 3, 1. Online unter: https://vcoe.at/files/vcoe/uploads/Magazin/2019/2019-03\%20-\%20Energieewende\%20Adieu\%20Erdoel/ VCO $\%$ CC\%88-Magazin\%202019-03\%20Adieu\%20Erdoel\%20 Die\%20Energiezukunft\%20ist\%20erneuerbar.pdf (letzter Zugriff: 31.05.2020).

Vetter, A., Chrischilles, E., Eisenack, K., Kind, C., Mahrenholz, P. \& Pechan, A. (2017) Anpassung an den Klimawandel als neues Politikfeld. In: Brasseur, G.P., Jacob, D. \& Schuck-Zöller, S. (Hrsg.) Klimawandel in Deutschland: Entwicklung, Folgen, Risiken und Perspektiven, S. 325-334. Springer Spektrum. Berlin, Deutschland. DOI: https://doi.org/10.1007/978-3-662-50397-3_32

Webb, T.L., Sniehotta, F.F. \& Michie, S. (2010) Using theories of behaviour change to inform interventions for addictive behavio- 
urs. Addiction 105(11), 1879-1892. DOI: https://doi.org/10.1111/ j.1360-0443.2010.03028.x

Windsperger, A., Windsperger, B., Bird, D.N., Jungmeier, G., Schwaiger, H., Frischknecht, R., Nathani, C., Guhsl, R. \& Buchegger, A. (2017) Life cycle based modelling of greenhouse gas emissions of Austrian consumption. Publizierbarer Endbericht zum Projekt climAconsum, gefördert durch den Klima- und Energiefonds im Rahmen des Programms „Austrian Climate Research Programme“ (ACRP). Online unter: http://www.indoek.at/downloads/News_2018_climAconsum Endbericht.pdf (letzter Zugriff: 31.05.2020).

Wirth, H. (2019) Aktuelle Fakten zur Photovoltaik in Deutschland. Fraunhofer-Institut für Solare Energiesysteme ISE, Freiburg, Deutschland. Online unter: https://www.ise.fraunhofer.de/content/ dam/ise/de/documents/publications/studies/aktuelle-fakten-zur-photovoltaik-in-deutschland.pdf (letzter Zugriff: 31.05.2020).

Zeithaml, V.A. \& Bitner, M.J. (1996) Services marketing: integrating customer focus across the firm. McGraw-Hill, New York, NY, USA.

Open Access Dieses Buch wird unter der Creative Commons Namensnennung 4.0 International Lizenz (http://creativecommons.org/licenses/by/4.0/deed.de) veröffentlicht, welche die Nutzung, Vervielfältigung, Bearbeitung, Verbreitung und Wiedergabe in jeglichem Medium und Format erlaubt, sofern Sie den/die ursprünglichen Autor(en) und die Quelle ordnungsgemäß nennen, einen Link zur Creative Commons Lizenz beifügen und angeben, ob Änderungen vorgenommen wurden.

Die in diesem Buch enthaltenen Bilder und sonstiges Drittmaterial unterliegen ebenfalls der genannten Creative Commons Lizenz, sofern sich aus der Abbildungslegende nichts anderes ergibt. Sofern das betreffende Material nicht unter der genannten Creative Commons Lizenz steht und die betreffende Handlung nicht nach gesetzlichen Vorschriften erlaubt ist, ist für die oben aufgeführten Weiterverwendungen des Materials die Einwilligung des jeweiligen Rechteinhabers einzuholen. 